\title{
Thermophysical and thermodynamic properties of ionic liquids over an extended pressure range: $[\mathrm{bmim}]\left[\mathrm{NTf}_{2}\right]$ and $[\mathrm{hmim}]\left[\mathrm{NTf}_{2}\right]$ is
}

\author{
R. Gomes de Azevedo ${ }^{\text {a }}$, J.M.S.S. Esperança ${ }^{\text {a }}$, J. Szydlowski a,b, Z.P. Visak ${ }^{\text {a }}$, \\ P.F. Pires ${ }^{a}$, H.J.R. Guedes ${ }^{\text {a,c }}$, L.P.N. Rebelo ${ }^{\mathrm{a}, *}$ \\ a Instituto de Tecnologia Química e Biológica, ITQB 2, Universidade Nova de Lisboa, Av.República, Apartado 127, 2780-901 Oeiras, Portugal \\ ${ }^{\mathrm{b}}$ Chemistry Department, Warsaw University, Zwirki I Wigury 101, 02-089 Warsaw, Poland \\ ${ }^{\mathrm{c}}$ REQUIMTE, Departamento de Química, Faculdade de Ciências e Tecnologia, Universidade Nova de Lisboa, 2829-516 Caparica, Portugal
}

Received 21 January 2005; received in revised form 20 April 2005; accepted 20 April 2005

Available online 27 June 2005

\begin{abstract}
The current study focuses on 1-butyl-3-methylimidazolium bis(trifluoromethylsulfonyl)amide, [bmim][NTf f $_{2}$, and 1-hexyl-3methylimidazolium bis(trifluoromethylsulfonyl)amide, $[\mathrm{hmim}]\left[\mathrm{NTf}_{2}\right]$. The objective is to study the influence of pressure as well as that of the cation's alkyl chain length on several properties of this type of ionic liquids. Speed of propagation of ultrasound waves and densities in pure ionic liquids (ILs) as a function of temperature and pressure have been determined. Several other thermodynamic properties such as compressibilities, expansivities and heat capacities have been obtained. Speed of sound measurements have been carried out in broad ranges of temperature $(283<T / \mathrm{K}<323)$ and pressure $(0.1<p / \mathrm{MPa}<150)$, using a non-intrusive microcell. Density measurements have been performed at broad ranges of temperature $(298<T / \mathrm{K}<333)$ and pressure $(0.1<p / \mathrm{MPa}<60)$ using a vibrating tube densimeter. The pressure dependence of heat capacities, which is generally mild, is highly dependent on the curvature of the temperature dependence of density.
\end{abstract}

(c) 2005 Elsevier Ltd. All rights reserved.

Keywords: Speed of sound; Density; Heat capacity; Pressure; Ionic liquids; [bmim][ $\left.\mathrm{NTf}_{2}\right]$; $[\mathrm{hmim}]\left[\mathrm{NTf}_{2}\right]$

\section{Introduction}

Due to their combined low viscosity, broad temperature range of liquid stability, and absence of halogen atoms in potentially chemically reactive forms, the 1-alkyl-3-methyl imidazolium bis(trifluoromethylsulfonyl)amide, $\left[\mathrm{C}_{n} \mathrm{~min}\right]\left[\mathrm{NTf}_{2}\right]$, class of ionic liquids (ILs) is one of the most promising to become important in diverse applications.

\footnotetext{
This paper was comissioned as part of the special issue, IONIC LIQUIDS, published in issue $37 / 6$ of the journal.

${ }^{*}$ Corresponding author. Tel.: +351 214469 441; fax: +351214411 277.

E-mail address: luis.rebelo@itqb.unl.pt (L.P.N. Rebelo).
}

The increasing attention given to ionic liquids as possible replacement solvents for volatile organic compounds is driving their research towards the development of a sustainable green chemistry, guiding efforts in this field in the direction of new, harmless substances. Unfortunately, the thermophysical characterization of benign ILs is by no means extensive. Examples of very recent reviews and systematic studies can be found, for instance, in references [1-4] and references therein. They show that some studies are still directed to compounds such as $\left[\mathrm{C}_{n} \min \right]\left[\mathrm{PF}_{6}\right]$ or $\left[\mathrm{C}_{n} \min \right]\left[\mathrm{BF}_{4}\right]$, that, despite their historical and fundamental importance, are known to easily produce $\mathrm{HF}$ and other aggressive chemicals at moderate and high temperatures. We have thus chosen to embark on the study of thermophysical and thermodynamic properties of a possibly cleaner alternative, 
namely the series containing the popular cation $1-\mathrm{C}_{n}-3-$ methylimidazolium combined with the anion bis(trifluoro methylsulfonyl)amide, $\left[\mathrm{N}\left\{\mathrm{SO}_{2}\left(\mathrm{CF}_{3}\right)\right\}_{2}\right]^{-}$.

The speed of sound, $u$, is a property that can be experimentally determined with great precision over a broad range of temperature and pressure. Since $u$ can be related to the first pressure partial derivative of density, accurate sound-speed data can be used to enhance the development of equations of state. Furthermore, it is a very useful source of information for computing values of other thermodynamic properties which are difficult to obtain at extreme experimental conditions, such as calorimetric data at high pressures. One possible method for deriving a wealth of thermodynamic properties consists in the measurement of the whole $(p, \rho, T)$ and $(p, u, T)$ surfaces in order to obtain by differentiation the derived properties of the investigated compound. This method has proven useful, for instance, in the calculation of derived properties of protonated and deuterated acetone [5,6]. Nonetheless, commonly accepted as a more reliable method for deriving thermodynamic properties from speed of sound data is that which allows, by integration, the calculation of $(p, \rho, T)$ and $\left(p C_{p}, T\right)$ surfaces from merely one isobar of both density and isobaric heat capacity [7-10]. This method allows one to calculate other thermophysical properties such as isentropic $\left(\kappa_{S}\right)$ and isothermal $\left(\kappa_{T}\right)$ compressibilities, isobaric thermal expansivities $\left(\alpha_{p}\right)$, isobaric $\left(C_{p}\right)$ and isochoric $\left(C_{v}\right)$ heat capacities and thermal pressure coefficients $\left(\gamma_{v}\right)$ over the entire range of pressures of the speed of sound measurements.

\section{Experimental}

\subsection{Acoustic cell and densimeter}

Measurements of the speed of sound were performed in the temperature range $283<T / \mathrm{K}<323$ and pressure

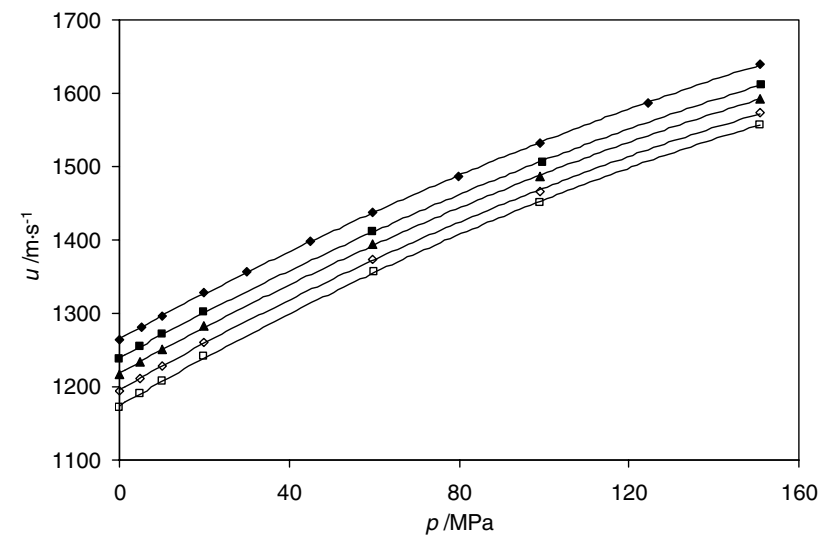

FIGURE 1. Isotherms of the experimental speed of sound of [bmim][NTf $\left.{ }_{2}\right] . \diamond, 283.15 \mathrm{~K} ; \mathbf{\square}, 293.15 \mathrm{~K} ; \boldsymbol{\Delta}, 303.15 \mathrm{~K} ; \diamond, 313.15 \mathrm{~K}$; $\square, 323.15 \mathrm{~K}$. range $0.1<p / \mathrm{MPa}<150$. The speed of propagation of sound waves in liquids was determined using a novel micro-cell (internal volume $\sim 0.8 \cdot 10^{-6} \mathrm{~m}^{3}$ ) and a nonintrusive method at an operational frequency of 0.5 MHz. We used the same experimental set-up as previously described in great detail elsewhere [5]. Pressure is measured using an Omega pressure transducer, which was calibrated against a high-accuracy Heise gauge. Pressure precision and accuracy are better than $\pm 0.05 \%$. The acoustic cell is placed in a Hart Scientific calibration bath (stability $\pm 0.001 \mathrm{~K}$ ) and temperature

TABLE 1

Experimental speed of sound, $u$, data for $[\mathrm{bmim}]\left[\mathrm{NTf}_{2}\right]$ as a function of temperature, $T$, and pressure, $p$

\begin{tabular}{|c|c|c|}
\hline$p / \mathrm{MPa}$ & & $u /\left(\mathrm{m} \cdot \mathrm{s}^{-1}\right)$ \\
\hline \multicolumn{3}{|c|}{$T=283.15 \mathrm{~K}$} \\
\hline 0.10 & & 1264.55 \\
\hline 5.09 & & 1280.80 \\
\hline 9.98 & & 1296.79 \\
\hline 20.02 & & 1327.89 \\
\hline 29.95 & & 1357.26 \\
\hline 44.83 & & 1398.70 \\
\hline 59.71 & & 1437.55 \\
\hline 79.70 & & 1486.76 \\
\hline 98.92 & & 1531.26 \\
\hline 124.41 & & 1586.58 \\
\hline 151.00 & & 1640.47 \\
\hline \multicolumn{3}{|c|}{$T=293.15 \mathrm{~K}$} \\
\hline 0.10 & & 1238.06 \\
\hline 5.00 & & 1254.63 \\
\hline 10.02 & & 1271.15 \\
\hline 20.01 & & 1302.51 \\
\hline 59.65 & & 1411.77 \\
\hline 99.72 & & 1505.90 \\
\hline 151.20 & & 1611.94 \\
\hline \multicolumn{3}{|c|}{$T=303.15 \mathrm{~K}$} \\
\hline 0.10 & & 1217.51 \\
\hline 5.03 & & 1233.96 \\
\hline 10.00 & & 1250.67 \\
\hline 20.03 & & 1282.84 \\
\hline 59.72 & & 1393.47 \\
\hline 98.96 & & 1485.86 \\
\hline 150.84 & & 1592.89 \\
\hline \multicolumn{3}{|c|}{$T=313.15 \mathrm{~K}$} \\
\hline 0.10 & & 1194.50 \\
\hline 5.01 & & 1211.49 \\
\hline 10.01 & & 1228.65 \\
\hline 19.96 & & 1261.10 \\
\hline 59.73 & & 1373.46 \\
\hline 98.97 & & 1466.37 \\
\hline 150.89 & & 1573.23 \\
\hline \multicolumn{3}{|c|}{$T=323.15 \mathrm{~K}$} \\
\hline 0.10 & & 1172.58 \\
\hline 5.05 & & 1190.18 \\
\hline 10.03 & & 1207.77 \\
\hline 19.94 & & 1240.95 \\
\hline 59.81 & & 1355.93 \\
\hline 99.22 & & 1450.79 \\
\hline 150.84 & & 1557.39 \\
\hline
\end{tabular}


is measured by a 4-wire platinum resistance thermometer (PRT) coupled to a Keithley digital multimeter (Model DMM 199). The PRT was previously calibrated, thus allowing temperature measurements on the ITS-90 scale with an estimated uncertainty better than $\pm 0.01 \mathrm{~K}$. Typically, the accuracy and precision of the sound-speed measurements are $\pm 0.2 \%$ and $\pm 0.05 \%$, respectively. Likewise, densities were measured using an Anton Paar DMA 512P densimeter [5] in the temperature range (298 to 333$) \mathrm{K}$ and pressure range ( 0.1 to 60$) \mathrm{MPa}$. The density overall uncertainty is estimated to be better than $0.02 \%$.

TABLE 2

Coefficients of equation (1) for $T / \mathrm{K}, p / \mathrm{MPa}$, and $u /\left(\mathrm{m} \cdot \mathrm{s}^{-1}\right)$, within the interval $(283<T / \mathrm{K}<323 ; 0.1<p / \mathrm{MPa}<150)$

\begin{tabular}{llrr}
\hline$a_{i j}$ & $j$ & \\
\cline { 2 - 4 }$i$ & 0 & 1 & \multicolumn{1}{l}{2} \\
\hline 0 & $1.66933 \cdot 10^{3}$ & -8.76289 & $9.53079 \cdot 10^{-3}$ \\
1 & $-4.57105 \cdot 10^{-1}$ & $2.07932 \cdot 10^{-2}$ & $-7.23757 \cdot 10^{-5}$ \\
2 & $-5.41339 \cdot 10^{-2}$ & $3.52903 \cdot 10^{-4}$ & $-5.75361 \cdot 10^{-7}$ \\
& & & \\
$b_{k l}$ & $l$ & & 2 \\
$k$ & 0 & 1 & $3.23167 \cdot 10^{-6}$ \\
\hline 0 & 1.00000 & $-4.58017 \cdot 10^{-3}$ & $-6.75836 \cdot 10^{-8}$ \\
1 & $-3.10366 \cdot 10^{-3}$ & $2.94127 \cdot 10^{-5}$ & $-2.59119 \cdot 10^{-10}$ \\
2 & $-2.59978 \cdot 10^{-5}$ & $1.65030 \cdot 10^{-7}$ & \\
\hline
\end{tabular}

\subsection{Chemicals}

[bmim] $\left[\mathrm{NTf}_{2}\right]$ and $[\mathrm{hmim}]\left[\mathrm{NTf}_{2}\right]$ were synthesized and purified at the QUILL Centre, Belfast, according to procedures found elsewhere [11]. They were washed several times with water to decrease the chloride content. It was checked that no precipitation (of $\mathrm{AgCl}$ ) would occur by addition of $\mathrm{AgNO}_{3}$ to the wash water. In order to reduce the water content and volatile compounds to negligible values, vacuum $(0.1 \mathrm{~Pa})$ and moderate temperature $\left(70{ }^{\circ} \mathrm{C}\right)$ were applied to the $[\mathrm{bmim}]\left[\mathrm{NTf}_{2}\right]$ and $[\mathrm{hmim}]\left[\mathrm{NTf}_{2}\right]$ samples for several days always

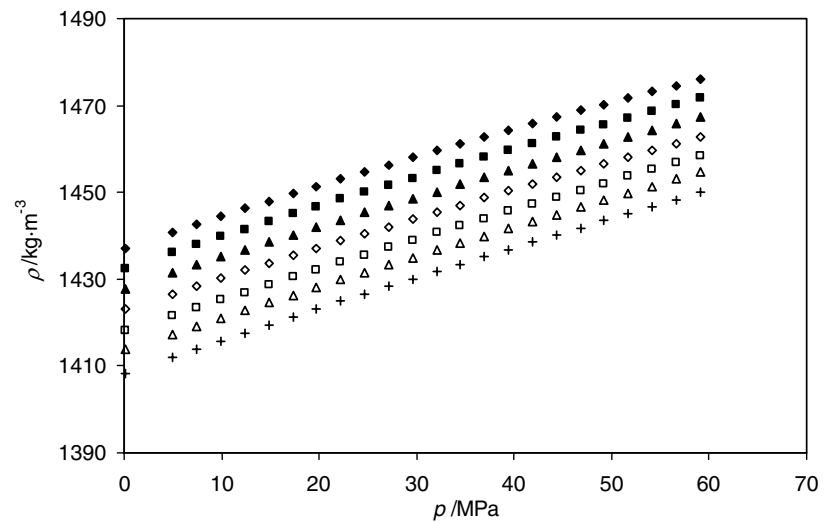

FIGURE 2. Isotherms of the experimental density of [bmim][ $\left.\mathrm{NTf}_{2}\right]$. $\diamond, 298.15 \mathrm{~K} ; \mathbf{\square}, 303.14 \mathrm{~K} ; \boldsymbol{\Delta}, 308.09 \mathrm{~K} ; \diamond, 313.15 \mathrm{~K} ; \square, 318.14 \mathrm{~K} ; \triangle$, $323.14 \mathrm{~K} ;+, 328.20 \mathrm{~K}$.

TABLE 3

Experimental density, $\rho /\left(\mathrm{kg} \cdot \mathrm{m}^{-3}\right)$, data for $[\mathrm{bmim}]\left[\mathrm{NTf}_{2}\right]$ as a function of temperature, $T$, and pressure, $p$

\begin{tabular}{|c|c|c|c|c|c|c|c|}
\hline \multirow[t]{2}{*}{$p / \mathrm{MPa}$} & \multicolumn{7}{|l|}{$T / \mathrm{K}$} \\
\hline & 298.15 & 303.14 & 308.09 & 313.15 & 318.14 & 323.14 & 328.20 \\
\hline 0.10 & 1437.04 & 1432.49 & 1427.92 & 1422.99 & 1418.06 & 1413.69 & 1408.32 \\
\hline 5.00 & 1440.78 & 1436.13 & 1431.50 & 1426.56 & 1421.63 & 1417.31 & 1412.04 \\
\hline 7.46 & 1442.64 & 1437.93 & 1433.28 & 1428.40 & 1423.41 & 1419.14 & 1413.97 \\
\hline 9.92 & 1444.45 & 1439.73 & 1435.07 & 1430.14 & 1425.23 & 1420.97 & 1415.75 \\
\hline 12.38 & 1446.22 & 1441.51 & 1436.81 & 1431.97 & 1427.00 & 1422.80 & 1417.61 \\
\hline 14.84 & 1447.99 & 1443.23 & 1438.55 & 1433.70 & 1428.76 & 1424.57 & 1419.44 \\
\hline 17.30 & 1449.71 & 1444.96 & 1440.27 & 1435.41 & 1430.50 & 1426.35 & 1421.25 \\
\hline 19.76 & 1451.41 & 1446.65 & 1441.96 & 1437.10 & 1432.21 & 1428.11 & 1423.05 \\
\hline 22.21 & 1453.09 & 1448.37 & 1443.64 & 1438.80 & 1433.93 & 1429.82 & 1424.84 \\
\hline 24.67 & 1454.75 & 1449.99 & 1445.32 & 1440.50 & 1435.62 & 1431.56 & 1426.58 \\
\hline 27.13 & 1456.40 & 1451.64 & 1446.94 & 1442.16 & 1437.31 & 1433.26 & 1428.34 \\
\hline 29.59 & 1458.04 & 1453.25 & 1448.61 & 1443.80 & 1439.01 & 1434.96 & 1430.04 \\
\hline 32.05 & 1459.61 & 1454.87 & 1450.21 & 1445.44 & 1440.66 & 1436.63 & 1431.76 \\
\hline 34.51 & 1461.19 & 1456.47 & 1451.80 & 1447.05 & 1442.31 & 1438.32 & 1433.46 \\
\hline 36.97 & 1462.78 & 1458.05 & 1453.43 & 1448.67 & 1443.93 & 1439.98 & 1435.15 \\
\hline 39.43 & 1464.31 & 1459.60 & 1454.99 & 1450.26 & 1445.59 & 1441.63 & 1436.83 \\
\hline 41.88 & 1465.81 & 1461.17 & 1456.59 & 1451.88 & 1447.20 & 1443.28 & 1438.52 \\
\hline 44.34 & 1467.33 & 1462.67 & 1458.14 & 1453.41 & 1448.84 & 1444.91 & 1440.15 \\
\hline 46.80 & 1468.80 & 1464.20 & 1459.69 & 1455.01 & 1450.43 & 1446.54 & 1441.79 \\
\hline 49.26 & 1470.28 & 1465.69 & 1461.21 & 1456.51 & 1452.03 & 1448.18 & 1443.46 \\
\hline 51.72 & 1471.69 & 1467.16 & 1462.74 & 1458.07 & 1453.64 & 1449.79 & 1445.11 \\
\hline 54.18 & 1473.13 & 1468.67 & 1464.26 & 1459.64 & 1455.23 & 1451.40 & 1446.72 \\
\hline 56.64 & 1474.55 & 1470.14 & 1465.80 & 1461.15 & 1456.83 & 1453.01 & 1448.35 \\
\hline 59.10 & 1475.94 & 1471.59 & 1467.28 & 1462.71 & 1458.39 & 1454.62 & 1449.96 \\
\hline
\end{tabular}


TABLE 4

Coefficients of second order polynomial fits, $Y=A+B$. $(T / \mathrm{K})+C \cdot(T / \mathrm{K})^{2}$ to atmospheric isobars of $Y=\rho$ or $Y=C_{p}$

\begin{tabular}{lll}
\hline & $\rho /\left(\mathrm{kg} \cdot \mathrm{m}^{-3}\right)$ & $C_{p} /\left(\mathrm{J} \cdot \mathrm{K}^{-1} \cdot \mathrm{kg}^{-1}\right)$ \\
\hline$A$ & $1.59720 \cdot 10^{3}$ & $2.39992 \cdot 10^{3}$ \\
$B$ & $-1.59362 \cdot 10^{-1}$ & -7.68270 \\
$C$ & $-1.26695 \cdot 10^{-3}$ & $1.33104 \cdot 10^{-2}$ \\
\hline
\end{tabular}

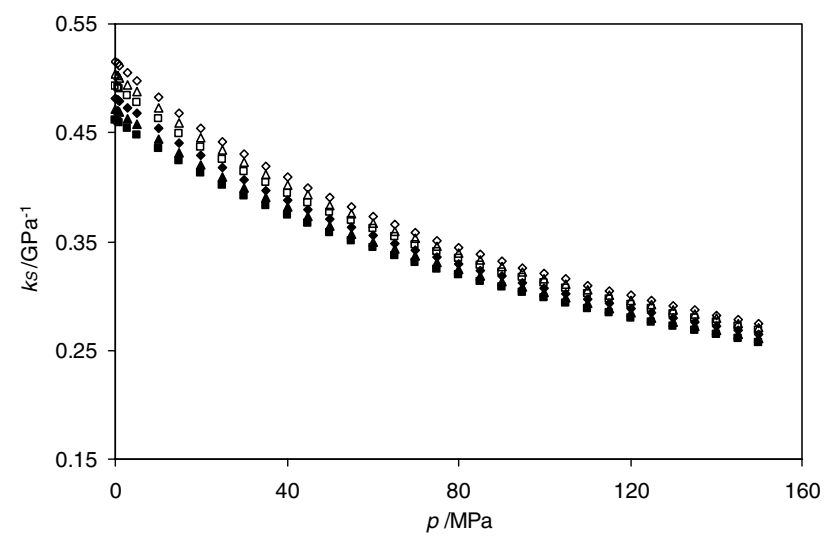

FIGURE 3. Isotherms for the isentropic compressibility of [bmim][NTf ${ }_{2}$. $\mathbf{\square}, 298.15 \mathrm{~K} ; \boldsymbol{\Delta}, 303.15 \mathrm{~K} ; \diamond, 308.15 \mathrm{~K} ; \square, 313.15 \mathrm{~K}$; $\triangle, 318.15 \mathrm{~K} ; \diamond, 323.15 \mathrm{~K}$.

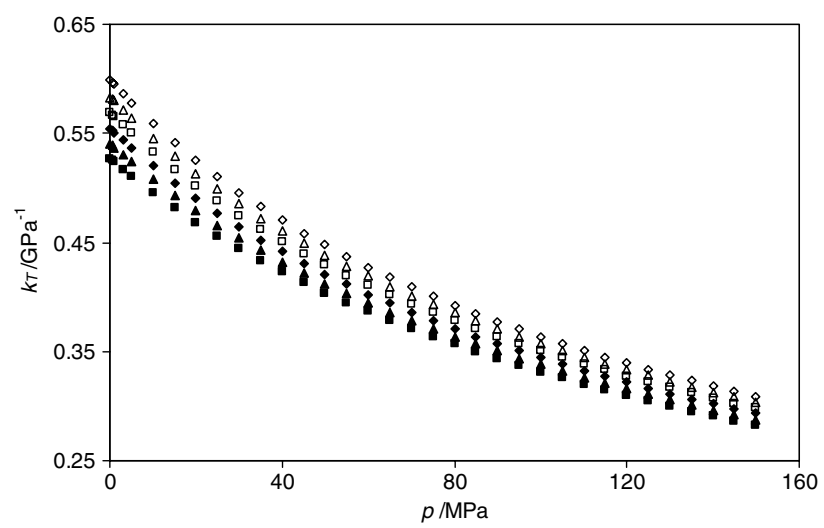

FIGURE 4. Isotherms for the isothermal compressibility of [bmim][NTf ${ }_{2}$. $\mathbf{\square}, 298.15 \mathrm{~K} ; \boldsymbol{\Delta}, 303.15 \mathrm{~K} ; \diamond, 308.15 \mathrm{~K} ; \square, 313.15 \mathrm{~K}$; $\triangle, 318.15 \mathrm{~K} ; \diamond, 323.15 \mathrm{~K}$.

immediately prior to their use. The samples were analysed by Karl-Fischer titration and showed a wt $\%$ of water lower than 75 ppm ("Crison" Karl-Fischer titration).

\section{Results and discussion}

\section{1. $[\mathrm{bmim}]\left[\mathrm{NTf}_{2}\right]$}

Sound-speed measurements have been carried out in a broad range of temperatures $(283<T / \mathrm{K}<323)$ and pressures $(0.1<p / \mathrm{MPa}<150)$. Figure 1 and table 1

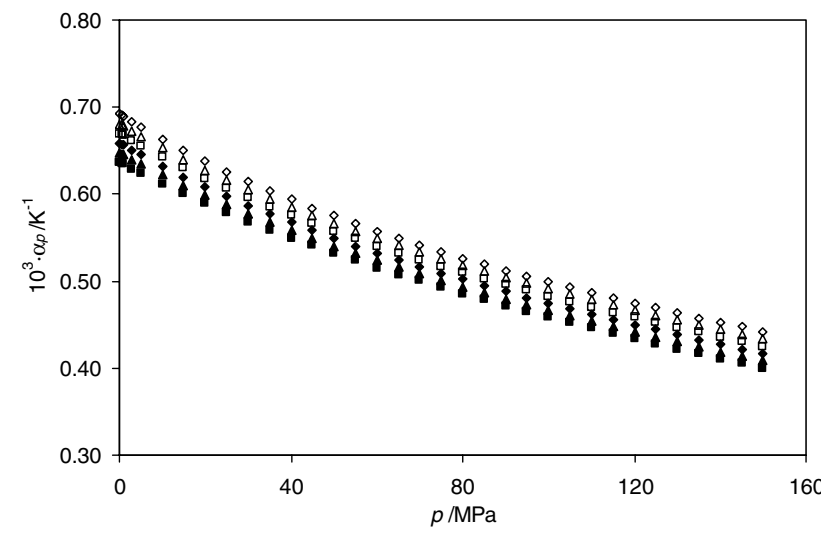

FIGURE 5. Isotherms for the isobaric expansivity of [bmim] $\left[\mathrm{NTf}_{2}\right]$. $\mathbf{\square}, 298.15 \mathrm{~K} ; \boldsymbol{\Delta}, 303.15 \mathrm{~K} ; \diamond, 308.15 \mathrm{~K} ; \square, 313.15 \mathrm{~K} ; \triangle, 318.15 \mathrm{~K} ; \diamond$, $323.15 \mathrm{~K}$.

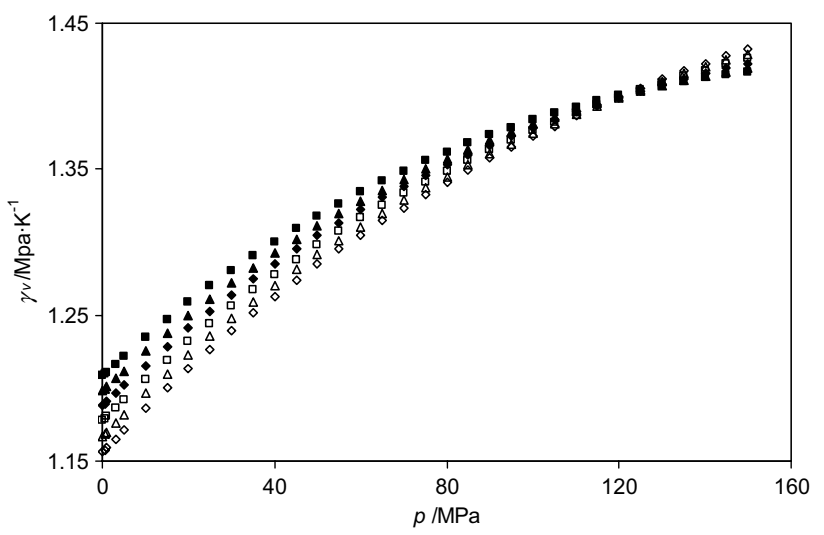

FIGURE 6. Isotherms for the thermal pressure coefficient of [bmim][NTf ${ }_{2}$ ]. $\mathbf{\square}, 298.15 \mathrm{~K} ; \boldsymbol{\Delta}, 303.15 \mathrm{~K} ; \diamond, 308.15 \mathrm{~K} ; \square, 313.15 \mathrm{~K}$; $\triangle, 318.15 \mathrm{~K} ; \diamond, 323.15 \mathrm{~K}$.

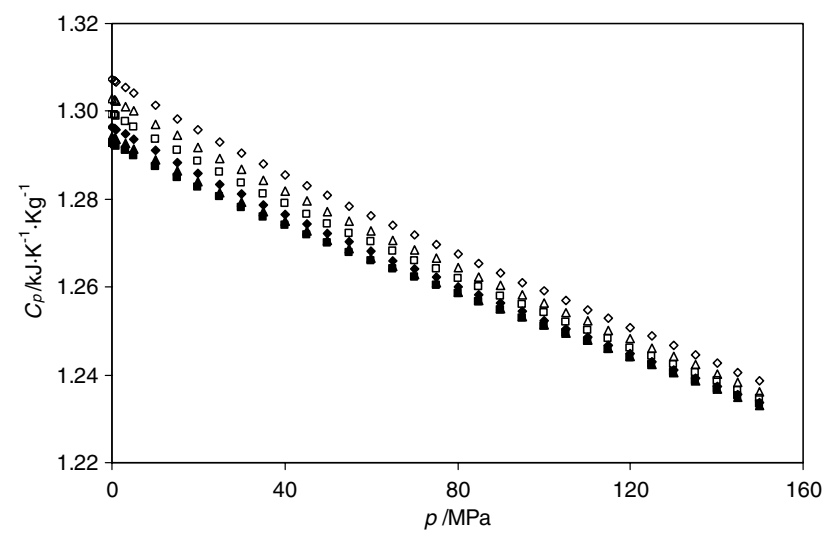

FIGURE 7. Isotherms for the isobaric heat capacity of [bmim][ $\left.\mathrm{NTf}_{2}\right]$. 口, $298.15 \mathrm{~K} ; \boldsymbol{\Delta}, 303.15 \mathrm{~K} ; \diamond, 308.15 \mathrm{~K} ; \square, 313.15 \mathrm{~K} ; \triangle$, $318.15 \mathrm{~K} ; \diamond$, $323.15 \mathrm{~K}$.

report the speed of sound behaviour as pressure and temperature change for $[\mathrm{bmim}]\left[\mathrm{NTf}_{2}\right]$. For the sake of economy, data are presented at nominal temperatures 
which, typically, differ from the actual ones by no more than $0.01 \mathrm{~K}$.

Original data have been fitted to a Pade $3 \times 3$ equation of the form

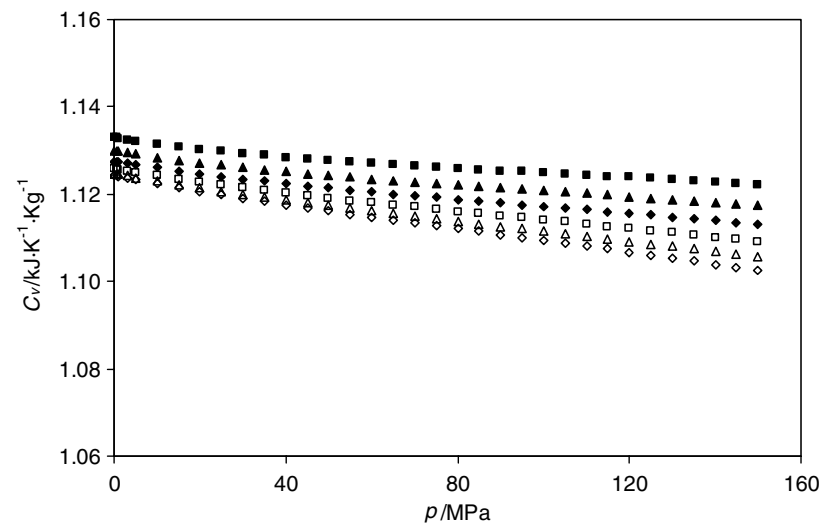

FIGURE 8. Isotherms for the isochoric heat capacity of [bmim][NTf $]$ ]. $\mathbf{\square}, 298.15 \mathrm{~K} ; \boldsymbol{\Delta}, 303.15 \mathrm{~K} ; \diamond, 308.15 \mathrm{~K} ; \square, 313.15 \mathrm{~K}$; $\triangle, 318.15 \mathrm{~K} ; \diamond, 323.15 \mathrm{~K}$.

TABLE 5

Calculated values of $\kappa_{S} /\left(\mathrm{GPa}^{-1}\right)$ for [bmim] $\left[\mathrm{NTf}_{2}\right]$

\begin{tabular}{|c|c|c|c|c|c|c|}
\hline \multirow[t]{2}{*}{$p / \mathrm{MPa}$} & \multicolumn{6}{|l|}{$T / \mathrm{K}$} \\
\hline & 298.15 & 303.15 & 308.15 & 313.15 & 318.15 & 323.15 \\
\hline 0.1 & $0.461_{7}$ & $0.471_{8}$ & $0.482_{0}$ & $0.492_{6}$ & $0.503_{5}$ & $0.514_{8}$ \\
\hline 0.5 & $0.460_{6}$ & $0.470_{6}$ & $0.480_{8}$ & $0.491_{3}$ & $0.502_{1}$ & $0.513_{4}$ \\
\hline 1 & $0.459_{2}$ & $0.469_{1}$ & $0.479_{2}$ & $0.489_{7}$ & $0.500_{5}$ & $0.511_{6}$ \\
\hline 3 & $0.453_{7}$ & $0.463_{3}$ & $0.473_{2}$ & $0.483_{4}$ & $0.493_{9}$ & $0.504_{8}$ \\
\hline 5 & $0.448_{3}$ & $0.457_{7}$ & $0.467_{4}$ & $0.477_{3}$ & $0.487_{5}$ & $0.498_{1}$ \\
\hline 10 & $0.435_{5}$ & $0.444_{5}$ & $0.453_{6}$ & $0.462_{9}$ & $0.472_{5}$ & $0.482_{4}$ \\
\hline 15 & $0.423_{6}$ & $0.432_{1}$ & $0.440_{7}$ & $0.449_{5}$ & $0.458_{6}$ & $0.467_{9}$ \\
\hline 20 & $0.412_{5}$ & $0.420_{6}$ & $0.428_{8}$ & $0.437_{1}$ & $0.445_{6}$ & $0.454_{4}$ \\
\hline 25 & $0.402_{1}$ & $0.409_{8}$ & $0.417_{6}$ & $0.425_{5}$ & $0.433_{6}$ & $0.441_{8}$ \\
\hline 30 & $0.392_{3}$ & $0.399_{7}$ & $0.407_{1}$ & $0.414_{6}$ & $0.422_{3}$ & $0.430_{1}$ \\
\hline 35 & $0.383_{1}$ & $0.390_{2}$ & $0.397_{2}$ & $0.404_{4}$ & $0.411_{7}$ & $0.419_{2}$ \\
\hline 40 & $0.374_{4}$ & $0.381_{2}$ & $0.388_{0}$ & $0.394_{8}$ & $0.401_{8}$ & $0.408_{9}$ \\
\hline 45 & $0.366_{2}$ & $0.372_{7}$ & $0.379_{2}$ & $0.385_{8}$ & $0.392_{4}$ & $0.399_{2}$ \\
\hline 50 & $0.358_{5}$ & $0.364_{7}$ & $0.371_{0}$ & $0.377_{3}$ & $0.383_{6}$ & $0.390_{0}$ \\
\hline 55 & $0.351_{1}$ & $0.357_{1}$ & $0.363_{1}$ & $0.369_{2}$ & $0.375_{3}$ & $0.381_{4}$ \\
\hline 60 & $0.344_{1}$ & $0.349_{9}$ & $0.355_{7}$ & $0.361_{5}$ & $0.367_{3}$ & $0.373_{2}$ \\
\hline 65 & $0.337_{4}$ & $0.343_{0}$ & $0.348_{6}$ & $0.354_{2}$ & $0.359_{8}$ & $0.365_{5}$ \\
\hline 70 & $0.331_{0}$ & $0.336_{5}$ & $0.341_{9}$ & $0.347_{3}$ & $0.352_{7}$ & $0.358_{1}$ \\
\hline 75 & $0.324_{9}$ & $0.330_{2}$ & $0.335_{5}$ & $0.340_{7}$ & $0.345_{9}$ & $0.351_{1}$ \\
\hline 80 & $0.319_{1}$ & $0.324_{2}$ & $0.329_{3}$ & $0.334_{4}$ & $0.339_{4}$ & $0.344_{4}$ \\
\hline 85 & $0.313_{5}$ & $0.318_{5}$ & $0.323_{4}$ & $0.328_{3}$ & $0.333_{2}$ & $0.338_{0}$ \\
\hline 90 & $0.308_{1}$ & $0.313_{0}$ & $0.317_{8}$ & $0.322_{5}$ & $0.327_{2}$ & $0.331_{9}$ \\
\hline 95 & $0.302_{9}$ & $0.307_{7}$ & $0.312_{4}$ & $0.317_{0}$ & $0.321_{6}$ & $0.326_{1}$ \\
\hline 100 & $0.298_{0}$ & $0.302_{7}$ & $0.307_{2}$ & $0.311_{7}$ & $0.316_{1}$ & $0.320_{5}$ \\
\hline 105 & $0.293_{2}$ & $0.297_{8}$ & $0.302_{2}$ & $0.306_{6}$ & $0.310_{8}$ & $0.315_{1}$ \\
\hline 110 & $0.288_{6}$ & $0.293_{1}$ & $0.297_{4}$ & $0.301_{6}$ & $0.305_{8}$ & $0.309_{9}$ \\
\hline 115 & $0.284_{2}$ & $0.288_{5}$ & $0.292_{8}$ & $0.296_{9}$ & $0.301_{0}$ & $0.305_{0}$ \\
\hline 120 & $0.279_{9}$ & $0.284_{2}$ & $0.288_{3}$ & $0.292_{3}$ & $0.296_{3}$ & $0.300_{2}$ \\
\hline 125 & $0.275_{7}$ & $0.279_{9}$ & $0.284_{0}$ & $0.287_{9}$ & $0.291_{8}$ & $0.295_{6}$ \\
\hline 130 & $0.271_{7}$ & $0.275_{8}$ & $0.279_{8}$ & $0.283_{6}$ & $0.287_{4}$ & $0.291_{1}$ \\
\hline 135 & $0.267_{9}$ & $0.271_{9}$ & $0.275_{7}$ & $0.279_{5}$ & $0.283_{2}$ & $0.286_{8}$ \\
\hline 140 & $0.264_{1}$ & $0.268_{0}$ & $0.271_{8}$ & $0.275_{5}$ & $0.279_{1}$ & $0.282_{6}$ \\
\hline 145 & $0.260_{4}$ & $0.264_{3}$ & $0.268_{0}$ & $0.271_{6}$ & $0.275_{2}$ & $0.278_{6}$ \\
\hline 150 & $0.256_{9}$ & $0.260_{7}$ & $0.264_{4}$ & $0.267_{9}$ & $0.271_{3}$ & $0.274_{7}$ \\
\hline
\end{tabular}

$$
\left(u / \mathrm{m} \cdot \mathrm{s}^{-1}\right)=\frac{\sum_{i=0}^{2} \sum_{j=0}^{2} a_{i j}(T / \mathrm{K})^{i}(p / \mathrm{MPa})^{j}}{\sum_{k=0}^{2} \sum_{l=0}^{2} b_{k l}(T / \mathrm{K})^{k}(p / \mathrm{MPa})^{l}} .
$$

The values of the coefficients were calculated by means of a least-squares analysis of the experimental results (using the algorithm of Marquardt-Levenberg) and are given in table 2. The standard deviation between the experimental and fitted values is found to be $0.05 \%$. This fitting equation was screened for 40 isotherms within the experimental temperature range $(283<T / \mathrm{K}<323)$, thus in steps of $1 \mathrm{~K}$, and for steps of $0.1 \mathrm{MPa}$ within the experimental interval of pressure $(0.1<p / \mathrm{MPa}<150)$ for a total of 61,500 data points. No poles for this rational function were found. Also, neither the temperature nor the pressure derivatives of $u(p, T)$ as described by equation (1) present any anomalies. Therefore, equation (1) can be safely used for interpolations.

TABLE 6

Calculated values of $\kappa_{T} /\left(\mathrm{GPa}^{-1}\right)$ for $[\mathrm{bmim}]\left[\mathrm{NTf}_{2}\right]$

\begin{tabular}{|c|c|c|c|c|c|c|}
\hline \multirow[t]{2}{*}{$p / \mathrm{MPa}$} & \multicolumn{6}{|l|}{$T / \mathrm{K}$} \\
\hline & 298.15 & 303.15 & 308.15 & 313.15 & 318.15 & 323.15 \\
\hline 0.1 & $0.526_{8}$ & $0.540_{3}$ & $0.554_{2}$ & $0.568_{5}$ & $0.583_{3}$ & $0.598_{6}$ \\
\hline 0.5 & $0.525_{4}$ & $0.538_{9}$ & $0.552_{7}$ & $0.566_{9}$ & $0.581_{6}$ & $0.596_{9}$ \\
\hline 1 & $0.523_{8}$ & $0.537_{1}$ & $0.550_{9}$ & $0.565_{0}$ & $0.579_{6}$ & $0.594_{7}$ \\
\hline 3 & $0.517_{1}$ & $0.530_{2}$ & $0.543_{6}$ & $0.557_{4}$ & $0.571_{7}$ & $0.586_{4}$ \\
\hline 5 & $0.510_{7}$ & $0.523_{5}$ & $0.536_{6}$ & $0.550_{1}$ & $0.564_{0}$ & $0.578_{3}$ \\
\hline 10 & $0.495_{5}$ & $0.507_{6}$ & $0.520_{0}$ & $0.532_{7}$ & $0.545_{8}$ & $0.559_{3}$ \\
\hline 15 & $0.481_{4}$ & $0.492_{9}$ & $0.504_{6}$ & $0.516_{6}$ & $0.529_{0}$ & $0.541_{7}$ \\
\hline 20 & $0.468_{1}$ & $0.479_{1}$ & $0.490_{2}$ & $0.501_{6}$ & $0.513_{3}$ & $0.525_{4}$ \\
\hline 25 & $0.455_{7}$ & $0.466_{2}$ & $0.476_{8}$ & $0.487_{7}$ & $0.498_{8}$ & $0.510_{2}$ \\
\hline 30 & $0.444_{1}$ & $0.454_{1}$ & $0.464_{2}$ & $0.474_{6}$ & $0.485_{2}$ & $0.496_{0}$ \\
\hline 35 & $0.433_{1}$ & $0.442_{7}$ & $0.452_{4}$ & $0.462_{3}$ & $0.472_{4}$ & $0.482_{7}$ \\
\hline 40 & $0.422_{7}$ & $0.432_{0}$ & $0.441_{3}$ & $0.450_{8}$ & $0.460_{4}$ & $0.470_{3}$ \\
\hline 45 & $0.412_{9}$ & $0.421_{8}$ & $0.430_{8}$ & $0.439_{9}$ & $0.449_{2}$ & $0.458_{6}$ \\
\hline 50 & $0.403_{7}$ & $0.412_{2}$ & $0.420_{9}$ & $0.429_{6}$ & $0.438_{5}$ & $0.447_{6}$ \\
\hline 55 & $0.394_{9}$ & $0.403_{2}$ & $0.411_{5}$ & $0.419_{9}$ & $0.428_{5}$ & $0.437_{1}$ \\
\hline 60 & $0.386_{5}$ & $0.394_{5}$ & $0.402_{6}$ & $0.410_{7}$ & $0.418_{9}$ & $0.427_{3}$ \\
\hline 65 & $0.378_{5}$ & $0.386_{3}$ & $0.394_{1}$ & $0.401_{9}$ & $0.409_{9}$ & $0.417_{9}$ \\
\hline 70 & $0.370_{9}$ & $0.378_{4}$ & $0.386_{0}$ & $0.393_{6}$ & $0.401_{3}$ & $0.409_{1}$ \\
\hline 75 & $0.363_{6}$ & $0.370_{9}$ & $0.378_{3}$ & $0.385_{7}$ & $0.393_{1}$ & $0.400_{6}$ \\
\hline 80 & $0.356_{6}$ & $0.363_{8}$ & $0.370_{9}$ & $0.378_{1}$ & $0.385_{3}$ & $0.392_{5}$ \\
\hline 85 & $0.350_{0}$ & $0.356_{9}$ & $0.363_{9}$ & $0.370_{8}$ & $0.377_{8}$ & $0.384_{9}$ \\
\hline 90 & $0.343_{5}$ & $0.350_{3}$ & $0.357_{1}$ & $0.363_{9}$ & $0.370_{7}$ & $0.377_{5}$ \\
\hline 95 & $0.337_{4}$ & $0.344_{0}$ & $0.350_{6}$ & $0.357_{2}$ & $0.363_{8}$ & $0.370_{5}$ \\
\hline 100 & $0.331_{5}$ & $0.338_{0}$ & $0.344_{4}$ & $0.350_{8}$ & $0.357_{2}$ & $0.363_{7}$ \\
\hline 105 & $0.325_{8}$ & $0.332_{1}$ & $0.338_{4}$ & $0.344_{7}$ & $0.350_{9}$ & $0.357_{2}$ \\
\hline 110 & $0.320_{3}$ & $0.326_{5}$ & $0.332_{6}$ & $0.338_{8}$ & $0.344_{9}$ & $0.351_{0}$ \\
\hline 115 & $0.315_{0}$ & $0.321_{0}$ & $0.327_{1}$ & $0.333_{1}$ & $0.339_{0}$ & $0.345_{0}$ \\
\hline 120 & $0.309_{8}$ & $0.315_{8}$ & $0.321_{7}$ & $0.327_{6}$ & $0.333_{4}$ & $0.339_{3}$ \\
\hline 125 & $0.304_{9}$ & $0.310_{7}$ & $0.316_{5}$ & $0.322_{3}$ & $0.328_{0}$ & $0.333_{7}$ \\
\hline 130 & $0.300_{1}$ & $0.305_{8}$ & $0.311_{5}$ & $0.317_{1}$ & $0.322_{7}$ & $0.328_{3}$ \\
\hline 135 & $0.295_{4}$ & $0.301_{1}$ & $0.306_{7}$ & $0.312_{2}$ & $0.317_{7}$ & $0.323_{2}$ \\
\hline 140 & $0.290_{9}$ & $0.296_{5}$ & $0.302_{0}$ & $0.307_{4}$ & $0.312_{8}$ & $0.318_{1}$ \\
\hline 145 & $0.286_{6}$ & $0.292_{0}$ & $0.297_{4}$ & $0.302_{7}$ & $0.308_{0}$ & $0.313_{3}$ \\
\hline 150 & $0.282_{3}$ & $0.287_{7}$ & $0.293_{0}$ & $0.298_{2}$ & $0.303_{4}$ & $0.308_{6}$ \\
\hline
\end{tabular}


Density measurements have been carried out at a broad range of temperatures $(298<T / \mathrm{K}<328)$ and pressures $(0.1<p / \mathrm{MPa}<60)$. The experimental data are presented both in figure 2 and table 3.

The use of the speed of sound data to derive other thermodynamic properties requires the knowledge of one isobar of both density and heat capacity. There are already two sets of heat capacity data available $[2,12]$ and several sets of atmospheric density data $[2,3,13,14]$. The choice of the heat capacity isobar for the thermodynamic properties calculations was based on the comparison of the experimental data available. The heat capacity data presented by Fredlake et al. [2] are higher than those presented by Holbrey et al. [12] (which may possibly be an indication of higher sample purity since it is quite uncommon to find typical impurities that are responsible for the rise of these properties).

The atmospheric isobars of both our current density data and the literature values of heat capacity [2] were fitted with a second order polynomial equation up to $328 \mathrm{~K}$. The coefficients of the fittings are shown in

TABLE 7

Calculated values of $\alpha_{p} /\left(\mathrm{K}^{-1}\right) \cdot 10^{3}$ for $[\mathrm{bmim}]\left[\mathrm{NTf}_{2}\right]$

\begin{tabular}{|c|c|c|c|c|c|c|}
\hline \multirow[t]{2}{*}{$p / \mathrm{MPa}$} & \multicolumn{6}{|l|}{$T / \mathrm{K}$} \\
\hline & 298.15 & 303.15 & 308.15 & 313.15 & 318.15 & 323.15 \\
\hline 0.1 & $0.636_{6}$ & $0.647_{5}$ & $0.658_{5}$ & $0.669_{6}$ & $0.680_{8}$ & $0.692_{1}$ \\
\hline 0.5 & $0.635_{6}$ & $0.646_{4}$ & $0.657_{4}$ & $0.668_{4}$ & $0.679_{6}$ & $0.690_{9}$ \\
\hline 1 & $0.634_{2}$ & $0.645_{1}$ & $0.656_{0}$ & $0.667_{0}$ & $0.678_{1}$ & $0.689_{3}$ \\
\hline 3 & $0.629_{1}$ & $0.639_{7}$ & $0.650_{5}$ & $0.661_{3}$ & $0.672_{2}$ & $0.683_{3}$ \\
\hline 5 & $0.624_{0}$ & $0.634_{5}$ & $0.645_{1}$ & $0.655_{8}$ & $0.666_{5}$ & $0.677_{4}$ \\
\hline 10 & $0.611_{8}$ & $0.621_{9}$ & $0.632_{2}$ & $0.642_{5}$ & $0.652_{9}$ & $0.663_{4}$ \\
\hline 15 & $0.600_{2}$ & $0.610_{0}$ & $0.619_{9}$ & $0.629_{9}$ & $0.640_{0}$ & $0.650_{2}$ \\
\hline 20 & $0.589_{2}$ & $0.598_{7}$ & $0.608_{3}$ & $0.618_{0}$ & $0.627_{8}$ & $0.637_{7}$ \\
\hline 25 & $0.578_{7}$ & $0.588_{0}$ & $0.597_{3}$ & $0.606_{8}$ & $0.616_{3}$ & $0.625_{9}$ \\
\hline 30 & $0.568_{6}$ & $0.577_{7}$ & $0.586_{8}$ & $0.596_{0}$ & $0.605_{3}$ & $0.614_{7}$ \\
\hline 35 & $0.558_{9}$ & $0.567_{8}$ & $0.576_{8}$ & $0.585_{8}$ & $0.594_{9}$ & $0.604_{1}$ \\
\hline 40 & $0.549_{7}$ & $0.558_{4}$ & $0.567_{1}$ & $0.576_{0}$ & $0.584_{9}$ & $0.593_{9}$ \\
\hline 45 & $0.540_{7}$ & $0.549_{3}$ & $0.557_{9}$ & $0.566_{6}$ & $0.575_{4}$ & $0.584_{2}$ \\
\hline 50 & $0.532_{1}$ & $0.540_{5}$ & $0.549_{0}$ & $0.557_{6}$ & $0.566_{3}$ & $0.575_{0}$ \\
\hline 55 & $0.523_{7}$ & $0.532_{1}$ & $0.540_{5}$ & $0.549_{0}$ & $0.557_{5}$ & $0.566_{1}$ \\
\hline 60 & $0.515_{7}$ & $0.523_{9}$ & $0.532_{2}$ & $0.540_{6}$ & $0.549_{1}$ & $0.557_{6}$ \\
\hline 65 & $0.507_{8}$ & $0.516_{0}$ & $0.524_{3}$ & $0.532_{6}$ & $0.540_{9}$ & $0.549_{4}$ \\
\hline 70 & $0.500_{3}$ & $0.508_{4}$ & $0.516_{6}$ & $0.524_{8}$ & $0.533_{1}$ & $0.541_{5}$ \\
\hline 75 & $0.492_{9}$ & $0.500_{9}$ & $0.509_{1}$ & $0.517_{3}$ & $0.525_{5}$ & $0.533_{8}$ \\
\hline 80 & $0.485_{7}$ & $0.493_{7}$ & $0.501_{8}$ & $0.510_{0}$ & $0.518_{2}$ & $0.526_{5}$ \\
\hline 85 & $0.478_{7}$ & $0.486_{7}$ & $0.494_{8}$ & $0.502_{9}$ & $0.511_{1}$ & $0.519_{3}$ \\
\hline 90 & $0.471_{8}$ & $0.479_{8}$ & $0.487_{9}$ & $0.496_{0}$ & $0.504_{2}$ & $0.512_{4}$ \\
\hline 95 & $0.465_{2}$ & $0.473_{2}$ & $0.481_{2}$ & $0.489_{3}$ & $0.497_{5}$ & $0.505_{7}$ \\
\hline 100 & $0.458_{6}$ & $0.466_{6}$ & $0.474_{7}$ & $0.482_{8}$ & $0.490_{9}$ & $0.499_{2}$ \\
\hline 105 & $0.452_{3}$ & $0.460_{3}$ & $0.468_{3}$ & $0.476_{4}$ & $0.484_{6}$ & $0.492_{8}$ \\
\hline 110 & $0.446_{0}$ & $0.454_{0}$ & $0.462_{1}$ & $0.470_{2}$ & $0.478_{4}$ & $0.486_{6}$ \\
\hline 115 & $0.439_{9}$ & $0.447_{9}$ & $0.456_{0}$ & $0.464_{2}$ & $0.472_{4}$ & $0.480_{6}$ \\
\hline 120 & $0.433_{9}$ & $0.441_{9}$ & $0.450_{1}$ & $0.458_{2}$ & $0.466_{5}$ & $0.474_{8}$ \\
\hline 125 & $0.428_{0}$ & $0.436_{1}$ & $0.444_{2}$ & $0.452_{4}$ & $0.460_{7}$ & $0.469_{0}$ \\
\hline 130 & $0.422_{2}$ & $0.430_{3}$ & $0.438_{5}$ & $0.446_{8}$ & $0.455_{1}$ & $0.463_{4}$ \\
\hline 135 & $0.416_{5}$ & $0.424_{7}$ & $0.432_{9}$ & $0.441_{2}$ & $0.449_{5}$ & $0.457_{9}$ \\
\hline 140 & $0.410_{9}$ & $0.419_{1}$ & $0.427_{4}$ & $0.435_{7}$ & $0.444_{1}$ & $0.452_{5}$ \\
\hline 145 & $0.405_{4}$ & $0.413_{7}$ & $0.422_{0}$ & $0.430_{4}$ & $0.438_{8}$ & $0.447_{3}$ \\
\hline 150 & $0.400_{0}$ & $0.408_{3}$ & $0.416_{7}$ & $0.425_{1}$ & $0.433_{6}$ & $0.442_{1}$ \\
\hline
\end{tabular}

table 4. The method used to derive other thermodynamic properties is based on well-established thermodynamic relations. The speed of sound, $u$, is directly related to the pressure derivative of the density, $\rho$, through equation (2), in which the subscript $S$ denotes the condition of constant entropy

$$
\left(\frac{\partial \rho}{\partial p}\right)_{S}=\frac{1}{u^{2}}
$$

This derivative is related to the isothermal pressure derivative and the isobaric temperature derivative of the density through equation (3), where $C_{p}$ is the specific heat capacity at constant pressure

$$
\left(\frac{\partial \rho}{\partial p}\right)_{S}=\left(\frac{\partial \rho}{\partial p}\right)_{T}-\frac{T}{\rho^{2} \cdot C_{p}}\left(\frac{\partial \rho}{\partial T}\right)_{p}^{2}
$$

Rearranging the last equation and combining it with equation (2) creates equation (4), which also incorporates the definition of the thermal expansion coefficient, $\alpha_{p}$

TABLE 8

\begin{tabular}{|c|c|c|c|c|c|c|}
\hline \multirow[t]{2}{*}{$p / \mathrm{MPa}$} & \multicolumn{6}{|l|}{$T / \mathrm{K}$} \\
\hline & 298.15 & 303.15 & 308.15 & 313.15 & 318.15 & 323.15 \\
\hline 0.1 & $1.20_{8}$ & $1.19_{8}$ & $1.18_{8}$ & $1.17_{8}$ & $1.16_{7}$ & $1.15_{6}$ \\
\hline 0.5 & $1.21_{0}$ & $1.19_{9}$ & $1.18_{9}$ & $1.17_{9}$ & $1.16_{8}$ & $1.15_{7}$ \\
\hline 1 & $1.21_{1}$ & $1.20_{1}$ & $1.19_{1}$ & $1.18_{0}$ & $1.17_{0}$ & $1.15_{9}$ \\
\hline 3 & $1.21_{6}$ & $1.20_{6}$ & $1.19_{6}$ & $1.18_{6}$ & $1.17_{6}$ & $1.16_{5}$ \\
\hline 5 & $1.22_{2}$ & $1.21_{2}$ & $1.20_{2}$ & $1.19_{2}$ & $1.18_{2}$ & $1.17_{1}$ \\
\hline 10 & $1.23_{5}$ & $1.22_{5}$ & $1.21_{6}$ & $1.20_{6}$ & $1.19_{6}$ & $1.18_{6}$ \\
\hline 15 & $1.24_{7}$ & $1.23_{8}$ & 1.229 & $1.21_{9}$ & $1.21_{0}$ & $1.20_{0}$ \\
\hline 20 & $1.25_{9}$ & $1.25_{0}$ & $1.24_{1}$ & $1.23_{2}$ & $1.22_{3}$ & $1.21_{4}$ \\
\hline 25 & $1.27_{0}$ & $1.26_{1}$ & $1.25_{3}$ & $1.24_{4}$ & $1.23_{6}$ & $1.22_{7}$ \\
\hline 30 & $1.28_{0}$ & $1.27_{2}$ & $1.26_{4}$ & $1.25_{6}$ & $1.24_{8}$ & $1.23_{9}$ \\
\hline 35 & $1.29_{1}$ & 1.283 & $1.27_{5}$ & $1.26_{7}$ & $1.25_{9}$ & $1.25_{1}$ \\
\hline 40 & $1.30_{0}$ & $1.29_{3}$ & $1.28_{5}$ & $1.27_{8}$ & $1.27_{0}$ & $1.26_{3}$ \\
\hline 45 & $1.30_{9}$ & $1.30_{2}$ & $1.29_{5}$ & $1.28_{8}$ & $1.28_{1}$ & $1.27_{4}$ \\
\hline 50 & $1.31_{8}$ & $1.31_{1}$ & $1.30_{4}$ & $1.29_{8}$ & $1.29_{1}$ & $1.28_{5}$ \\
\hline 55 & $1.32_{6}$ & $1.32_{0}$ & $1.31_{3}$ & $1.30_{7}$ & $1.30_{1}$ & $1.29_{5}$ \\
\hline 60 & $1.33_{4}$ & $1.32_{8}$ & $1.32_{2}$ & $1.31_{6}$ & $1.31_{1}$ & $1.30_{5}$ \\
\hline 65 & $1.34_{2}$ & $1.33_{6}$ & $1.33_{0}$ & $1.32_{5}$ & $1.32_{0}$ & $1.31_{4}$ \\
\hline 70 & $1.34_{9}$ & $1.34_{3}$ & $1.33_{8}$ & $1.33_{3}$ & $1.32_{8}$ & $1.32_{4}$ \\
\hline 75 & $1.35_{6}$ & $1.35_{0}$ & $1.34_{6}$ & $1.34_{1}$ & $1.33_{7}$ & $1.33_{3}$ \\
\hline 80 & $1.36_{2}$ & $1.35_{7}$ & $1.35_{3}$ & $1.34_{9}$ & $1.34_{5}$ & $1.34_{1}$ \\
\hline 85 & $1.36_{8}$ & $1.36_{4}$ & $1.36_{0}$ & $1.35_{6}$ & $1.35_{3}$ & $1.34_{9}$ \\
\hline 90 & $1.37_{3}$ & $1.37_{0}$ & $1.36_{6}$ & $1.36_{3}$ & $1.36_{0}$ & $1.35_{7}$ \\
\hline 95 & $1.37_{9}$ & $1.37_{5}$ & $1.37_{2}$ & $1.37_{0}$ & $1.36_{7}$ & $1.36_{5}$ \\
\hline 100 & $1.38_{4}$ & $1.38_{1}$ & $1.37_{8}$ & $1.37_{6}$ & $1.37_{4}$ & $1.37_{2}$ \\
\hline 105 & $1.38_{8}$ & $1.38_{6}$ & $1.38_{4}$ & $1.38_{2}$ & $1.38_{1}$ & $1.38_{0}$ \\
\hline 110 & $1.39_{3}$ & $1.39_{1}$ & $1.38_{9}$ & $1.38_{8}$ & $1.38_{7}$ & $1.38_{6}$ \\
\hline 115 & $1.39_{7}$ & $1.39_{5}$ & $1.39_{4}$ & $1.39_{4}$ & $1.39_{3}$ & $1.39_{3}$ \\
\hline 120 & $1.40_{0}$ & 1.399 & 1.399 & 1.399 & 1.399 & $1.39_{9}$ \\
\hline 125 & $1.40_{4}$ & $1.40_{3}$ & $1.40_{3}$ & $1.40_{4}$ & $1.40_{5}$ & $1.40_{5}$ \\
\hline 130 & $1.40_{7}$ & $1.40_{7}$ & $1.40_{8}$ & $1.40_{9}$ & $1.41_{0}$ & $1.41_{1}$ \\
\hline 135 & $1.41_{0}$ & $1.41_{0}$ & $1.41_{2}$ & $1.41_{3}$ & $1.41_{5}$ & $1.41_{7}$ \\
\hline 140 & $1.41_{2}$ & $1.41_{4}$ & $1.41_{5}$ & $1.41_{8}$ & $1.42_{0}$ & $1.42_{2}$ \\
\hline 145 & $1.41_{5}$ & $1.41_{7}$ & $1.41_{9}$ & $1.42_{2}$ & $1.42_{5}$ & $1.42_{8}$ \\
\hline 150 & $1.41_{7}$ & $1.41_{9}$ & $1.42_{2}$ & $1.42_{5}$ & $1.42_{9}$ & $1.43_{3}$ \\
\hline
\end{tabular}

Calculated values of $\gamma_{v} /\left(\mathrm{MPa} \cdot \mathrm{K}^{-1}\right)$ for $[\mathrm{bmim}]\left[\mathrm{NTf}_{2}\right]$ 
$\left(\frac{\partial \rho}{\partial p}\right)_{T}=\frac{1}{u^{2}}+\frac{T}{C_{p}} \alpha_{p}^{2}$

$\alpha_{p}=-\frac{1}{\rho} \cdot\left(\frac{\partial \rho}{\partial T}\right)_{p}$.

It can also be shown that the pressure partial derivative of the isobaric heat capacity can be calculated with the following equation:

$$
\left(\frac{\partial C_{p}}{\partial p}\right)_{T}=-\frac{T}{\rho} \cdot\left[\alpha_{p}^{2}+\left(\frac{\partial \alpha_{p}}{\partial T}\right)_{p}\right] .
$$

Given an isobar of the density and of $C_{p}$, it is possible to integrate equations (4) and (6) over pressure [15] thus obtaining the $(p, \rho, T)$ and $\left(p, C_{p}, T\right)$ surfaces within the range of pressure and temperature of the experimental speed of sound data. The numerical integration procedure also allows one to calculate other properties, such as the isentropic compressibility, $\kappa_{S}$, the isothermal compressibility, $\kappa_{T}$, isobaric thermal expansivity, $\alpha_{p}$ the

TABLE 9

Calculated values of $C_{p} /\left(\mathrm{kJ} \cdot \mathrm{K}^{-1} \cdot \mathrm{kg}^{-1}\right)$ for $[\mathrm{bmim}]\left[\mathrm{NTf}_{2}\right]$

\begin{tabular}{|c|c|c|c|c|c|c|}
\hline \multirow[t]{2}{*}{$p / \mathrm{MPa}$} & \multicolumn{6}{|l|}{$T / \mathrm{K}$} \\
\hline & 298.15 & 303.15 & 308.15 & 313.15 & 318.15 & 323.15 \\
\hline 0.1 & $1.29_{3}$ & $1.29_{4}$ & $1.29_{6}$ & $1.29_{9}$ & $1.30_{3}$ & $1.30_{7}$ \\
\hline 0.5 & $1.29_{2}$ & $1.29_{4}$ & $1.29_{6}$ & $1.29_{9}$ & $1.30_{3}$ & $1.30_{7}$ \\
\hline 1 & $1.29_{2}$ & $1.29_{4}$ & $1.29_{6}$ & $1.29_{9}$ & $1.30_{2}$ & $1.30_{7}$ \\
\hline 3 & $1.29_{1}$ & 1.293 & $1.29_{5}$ & $1.29_{8}$ & $1.30_{1}$ & $1.30_{5}$ \\
\hline 5 & $1.29_{0}$ & $1.29_{1}$ & $1.29_{4}$ & $1.29_{7}$ & $1.30_{0}$ & $1.30_{4}$ \\
\hline 10 & $1.28_{7}$ & 1.289 & $1.29_{1}$ & $1.29_{4}$ & $1.29_{7}$ & $1.30_{1}$ \\
\hline 15 & $1.28_{5}$ & $1.28_{6}$ & $1.28_{8}$ & $1.29_{1}$ & $1.29_{4}$ & $1.29_{8}$ \\
\hline 20 & $1.28_{3}$ & $1.28_{4}$ & $1.28_{6}$ & $1.28_{8}$ & $1.29_{2}$ & $1.29_{6}$ \\
\hline 25 & $1.28_{0}$ & $1.28_{2}$ & $1.28_{3}$ & $1.28_{6}$ & $1.28_{9}$ & $1.29_{3}$ \\
\hline 30 & $1.27_{8}$ & $1.27_{9}$ & $1.28_{1}$ & $1.28_{4}$ & $1.28_{7}$ & $1.29_{0}$ \\
\hline 35 & $1.27_{6}$ & $1.27_{7}$ & $1.27_{9}$ & $1.28_{1}$ & $1.28_{4}$ & $1.28_{8}$ \\
\hline 40 & $1.27_{4}$ & $1.27_{5}$ & $1.27_{7}$ & $1.27_{9}$ & $1.28_{2}$ & $1.28_{5}$ \\
\hline 45 & $1.27_{2}$ & $1.27_{3}$ & $1.27_{4}$ & $1.27_{7}$ & $1.28_{0}$ & $1.28_{3}$ \\
\hline 50 & $1.27_{0}$ & $1.27_{1}$ & $1.27_{2}$ & $1.27_{4}$ & $1.27_{7}$ & $1.28_{1}$ \\
\hline 55 & $1.26_{8}$ & $1.26_{9}$ & $1.27_{0}$ & $1.27_{2}$ & $1.27_{5}$ & $1.27_{8}$ \\
\hline 60 & $1.26_{6}$ & $1.26_{7}$ & $1.26_{8}$ & $1.27_{0}$ & $1.27_{3}$ & $1.27_{6}$ \\
\hline 65 & $1.26_{4}$ & $1.26_{5}$ & $1.26_{6}$ & $1.26_{8}$ & $1.27_{1}$ & $1.27_{4}$ \\
\hline 70 & $1.26_{2}$ & $1.26_{3}$ & $1.26_{4}$ & $1.26_{6}$ & $1.26_{9}$ & $1.27_{2}$ \\
\hline 75 & $1.26_{0}$ & $1.26_{1}$ & $1.26_{2}$ & $1.26_{4}$ & $1.26_{6}$ & $1.27_{0}$ \\
\hline 80 & $1.25_{8}$ & $1.25_{9}$ & $1.26_{0}$ & $1.26_{2}$ & $1.26_{4}$ & $1.26_{7}$ \\
\hline 85 & $1.25_{7}$ & $1.25_{7}$ & $1.25_{8}$ & $1.26_{0}$ & $1.26_{2}$ & $1.26_{5}$ \\
\hline 90 & $1.25_{5}$ & $1.25_{5}$ & $1.25_{6}$ & $1.25_{8}$ & $1.26_{0}$ & $1.26_{3}$ \\
\hline 95 & $1.25_{3}$ & $1.25_{3}$ & $1.25_{4}$ & $1.25_{6}$ & $1.25_{8}$ & $1.26_{1}$ \\
\hline 100 & $1.25_{1}$ & $1.25_{1}$ & $1.25_{2}$ & $1.25_{4}$ & $1.25_{6}$ & $1.25_{9}$ \\
\hline 105 & $1.24_{9}$ & $1.25_{0}$ & $1.25_{1}$ & $1.25_{2}$ & $1.25_{4}$ & $1.25_{7}$ \\
\hline 110 & $1.24_{8}$ & $1.24_{8}$ & $1.24_{9}$ & $1.25_{0}$ & $1.25_{2}$ & $1.25_{5}$ \\
\hline 115 & $1.24_{6}$ & $1.24_{6}$ & $1.24_{7}$ & $1.24_{8}$ & $1.25_{0}$ & $1.25_{3}$ \\
\hline 120 & $1.24_{4}$ & $1.24_{4}$ & $1.24_{5}$ & $1.24_{6}$ & $1.24_{8}$ & $1.25_{1}$ \\
\hline 125 & $1.24_{2}$ & $1.24_{2}$ & $1.24_{3}$ & $1.24_{4}$ & $1.24_{6}$ & $1.24_{9}$ \\
\hline 130 & $1.24_{0}$ & $1.24_{0}$ & $1.24_{1}$ & $1.24_{2}$ & $1.24_{4}$ & $1.24_{7}$ \\
\hline 135 & $1.23_{9}$ & $1.23_{9}$ & $1.23_{9}$ & $1.24_{0}$ & $1.24_{2}$ & $1.24_{5}$ \\
\hline 140 & $1.23_{7}$ & $1.23_{7}$ & $1.23_{7}$ & $1.23_{9}$ & $1.24_{0}$ & $1.24_{3}$ \\
\hline 145 & $1.23_{5}$ & $1.23_{5}$ & $1.23_{5}$ & $1.23_{7}$ & $1.23_{8}$ & $1.24_{1}$ \\
\hline 150 & $1.23_{3}$ & $1.23_{3}$ & $1.23_{4}$ & $1.23_{5}$ & $1.23_{6}$ & $1.23_{9}$ \\
\hline
\end{tabular}

isochoric heat capacity $C_{v}$ and the thermal pressure coefficient, $\gamma_{v}$. The integration was carried out from atmospheric pressure up to $150 \mathrm{MPa}$. The results obtained for several thermodynamic properties are presented in figures 3 to 8 and tables 5 to 10 .

The comparison between the density data taken from the integration procedure and the experimental ones shows good agreement with a maximum deviation of about $0.1 \%$, even at pressures as high as $60 \mathrm{MPa}$. This deviation is small and can be ascribed to the uncertainty of the speed of sound measurements since this quantity is responsible for about $85 \%$ of the calculated $\mathrm{d} \rho / \mathrm{d} p$ in the integration process. At atmospheric pressure, our results are within the density interval estimated based on the number of significant digits presented for the fitting parameters given by Tokuda et al. [3], about $0.1 \%$ below those presented by Fredlake et al. [2], 0.07\% above the data point presented by Dzyuba and Bartsch [13], and $0.2 \%$ above the data point presented by Canongia Lopes et al. [14].
TABLE 10

Calculated values of $C_{v} /\left(\mathrm{kJ} \cdot \mathrm{K}^{-1} \cdot \mathrm{kg}^{-1}\right)$ for $[\mathrm{bmim}]\left[\mathrm{NTf}_{2}\right]$

\begin{tabular}{|c|c|c|c|c|c|c|}
\hline \multirow[t]{2}{*}{$p / \mathrm{MPa}$} & \multicolumn{6}{|l|}{$T / \mathrm{K}$} \\
\hline & 298.15 & 303.15 & 308.15 & 313.15 & 318.15 & 323.15 \\
\hline 0.1 & $1.13_{3}$ & $1.13_{0}$ & $1.12_{8}$ & $1.12_{6}$ & $1.12_{5}$ & $1.12_{4}$ \\
\hline 0.5 & $1.13_{3}$ & $1.13_{0}$ & $1.12_{7}$ & $1.12_{6}$ & $1.12_{5}$ & $1.12_{4}$ \\
\hline 1 & $1.13_{3}$ & $1.13_{0}$ & $1.12_{7}$ & $1.12_{6}$ & $1.12_{5}$ & $1.12_{4}$ \\
\hline 3 & $1.13_{2}$ & $1.12_{9}$ & $1.12_{7}$ & $1.12_{5}$ & $1.12_{4}$ & $1.12_{4}$ \\
\hline 5 & $1.13_{2}$ & $1.12_{9}$ & $1.12_{7}$ & $1.12_{5}$ & $1.12_{4}$ & $1.12_{3}$ \\
\hline 10 & $1.13_{2}$ & $1.12_{8}$ & $1.12_{6}$ & $1.12_{4}$ & $1.12_{3}$ & $1.12_{2}$ \\
\hline 15 & $1.13_{1}$ & $1.12_{8}$ & $1.12_{5}$ & $1.12_{3}$ & $1.12_{2}$ & $1.12_{1}$ \\
\hline 20 & $1.13_{0}$ & $1.12_{7}$ & $1.12_{5}$ & $1.12_{3}$ & $1.12_{1}$ & $1.12_{1}$ \\
\hline 25 & $1.13_{0}$ & $1.12_{7}$ & $1.12_{4}$ & $1.12_{2}$ & $1.12_{1}$ & $1.12_{0}$ \\
\hline 30 & $1.12_{9}$ & $1.12_{6}$ & $1.12_{3}$ & $1.12_{1}$ & $1.12_{0}$ & $1.11_{9}$ \\
\hline 35 & 1.129 & $1.12_{6}$ & $1.12_{3}$ & $1.12_{1}$ & $1.11_{9}$ & $1.11_{8}$ \\
\hline 40 & $1.12_{8}$ & $1.12_{5}$ & $1.12_{2}$ & $1.12_{0}$ & $1.11_{9}$ & $1.11_{8}$ \\
\hline 45 & $1.12_{8}$ & $1.12_{5}$ & $1.12_{2}$ & $1.12_{0}$ & $1.11_{8}$ & $1.11_{7}$ \\
\hline 50 & $1.12_{8}$ & $1.12_{4}$ & $1.12_{1}$ & $1.11_{9}$ & $1.11_{7}$ & $1.11_{6}$ \\
\hline 55 & $1.12_{7}$ & $1.12_{4}$ & $1.12_{1}$ & $1.11_{9}$ & $1.11_{7}$ & $1.11_{5}$ \\
\hline 60 & $1.12_{7}$ & $1.12_{3}$ & $1.12_{0}$ & $1.11_{8}$ & $1.11_{6}$ & $1.11_{5}$ \\
\hline 65 & $1.12_{7}$ & $1.12_{3}$ & $1.12_{0}$ & $1.11_{7}$ & $1.11_{6}$ & $1.11_{4}$ \\
\hline 70 & $1.12_{6}$ & $1.12_{3}$ & $1.12_{0}$ & $1.11_{7}$ & $1.11_{5}$ & $1.11_{3}$ \\
\hline 75 & $1.12_{6}$ & $1.12_{2}$ & $1.11_{9}$ & $1.11_{6}$ & $1.11_{4}$ & $1.11_{3}$ \\
\hline 80 & $1.12_{6}$ & $1.12_{2}$ & $1.11_{9}$ & $1.11_{6}$ & $1.11_{4}$ & $1.11_{2}$ \\
\hline 85 & $1.12_{6}$ & $1.12_{2}$ & $1.11_{8}$ & $1.11_{6}$ & $1.11_{3}$ & $1.11_{1}$ \\
\hline 90 & $1.12_{5}$ & $1.12_{1}$ & $1.11_{8}$ & $1.11_{5}$ & $1.11_{3}$ & $1.11_{1}$ \\
\hline 95 & $1.12_{5}$ & $1.12_{1}$ & $1.11_{8}$ & $1.11_{5}$ & $1.11_{2}$ & $1.11_{0}$ \\
\hline 100 & $1.12_{5}$ & $1.12_{1}$ & $1.11_{7}^{\circ}$ & $1.11_{4}$ & $1.11_{1}$ & $1.10_{9}$ \\
\hline 105 & $1.12_{5}$ & $1.12_{0}$ & $1.11_{7}$ & $1.11_{4}$ & $1.11_{1}$ & $1.10_{9}$ \\
\hline 110 & $1.12_{4}$ & $1.12_{0}$ & $1.11_{6}$ & $1.11_{3}$ & $1.11_{0}$ & $1.10_{8}$ \\
\hline 115 & $1.12_{4}$ & $1.12_{0}$ & $1.11_{6}$ & $1.11_{3}$ & $1.11_{0}$ & $1.10_{7}$ \\
\hline 120 & $1.12_{4}$ & $1.11_{9}$ & $1.11_{6}$ & $1.11_{2}$ & $1.10_{9}$ & $1.10_{7}$ \\
\hline 125 & $1.12_{4}$ & $1.11_{9}$ & $1.11_{5}$ & $1.11_{2}$ & $1.10_{9}$ & $1.10_{6}$ \\
\hline 130 & $1.12_{3}$ & $1.11_{9}$ & $1.11_{5}$ & $1.11_{1}$ & $1.10_{8}$ & $1.10_{5}$ \\
\hline 135 & $1.12_{3}$ & $1.11_{8}$ & $1.11_{4}$ & $1.11_{1}$ & $1.10_{7}$ & $1.10_{5}$ \\
\hline 140 & $1.12_{3}$ & $1.11_{8}$ & $1.11_{4}$ & $1.11_{0}$ & $1.10_{7}$ & $1.10_{4}$ \\
\hline 145 & $1.12_{3}$ & $1.11_{8}$ & $1.11_{3}$ & $1.11_{0}$ & $1.10_{6}$ & $1.10_{3}$ \\
\hline 150 & $1.12_{2}$ & $1.11_{7}$ & $1.11_{3}$ & $1.10_{9}$ & $1.10_{6}$ & $1.10_{3}$ \\
\hline
\end{tabular}




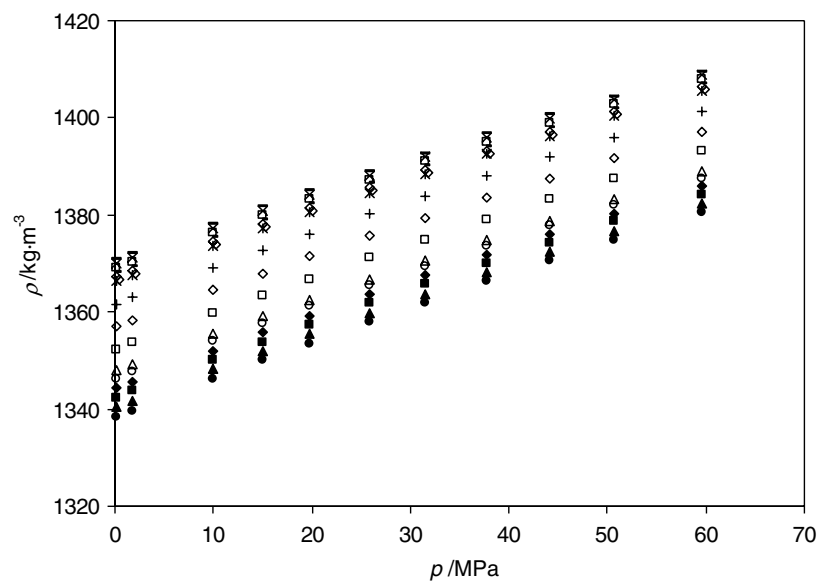

FIGURE 9. Isotherms of the experimental density of [hmim] $\left[\mathrm{NTf}_{2}\right]$.

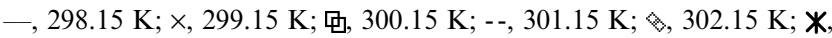
$303.15 \mathrm{~K} ;+$, $308.15 \mathrm{~K} ; \diamond, 313.15 \mathrm{~K} ; \square, 318.15 \mathrm{~K} ; \triangle, 323.15 \mathrm{~K} ; \bigcirc$, $325.15 \mathrm{~K} ; \bullet, 327.15 \mathrm{~K} ; \mathbf{\square}, 329.15 \mathrm{~K} ; \boldsymbol{\Delta}, 331.15 \mathrm{~K} ; \bullet$ ๑, $333.15 \mathrm{~K}$.

Compressibilities, as well as isobaric expansivities decrease markedly with pressure, according to figures 3 to 5 and tables 5 to 7 but they present a tendency to increase with increasing temperature at constant pressure.

The difference obtained on the derived properties, $\alpha_{p}$ and $\kappa_{T}$ when comparing the derivation of the measured densities with the integration method shows an average deviation of $5 \%$ and $-5 \%$, respectively. Although these
TABLE 12

Coefficients of the Tait equation, equation (7), for the density of $[\mathrm{hmim}]\left[\mathrm{NTf}_{2}\right]$ at each isotherm ${ }^{a}(0.1<p / \mathrm{MPa}<60)$

\begin{tabular}{lll}
\hline$T / \mathrm{K}$ & $A /\left(\mathrm{kg}^{-1} \cdot \mathrm{m}^{3}\right) \cdot 10^{5}$ & $B / \mathrm{MPa}$ \\
\hline 298.15 & -5.385 & 131.79 \\
299.15 & -5.633 & 138.56 \\
300.15 & -5.571 & 135.88 \\
301.15 & -5.809 & 142.42 \\
302.15 & -5.809 & 141.40 \\
303.15 & -6.075 & 148.72 \\
308.15 & -6.641 & 161.60 \\
313.15 & -7.375 & 178.70 \\
318.15 & -7.684 & 183.13 \\
323.15 & -8.249 & 197.44 \\
325.15 & -7.030 & 161.51 \\
327.15 & -7.838 & 180.24 \\
329.15 & -7.359 & 165.98 \\
331.15 & -7.473 & 167.85 \\
333.15 & -6.920 & 151.45 \\
\hline
\end{tabular}

${ }^{a}$ In the case of global fitting procedure one uses $A=4.42535$. $10^{-3}-2.79754 \cdot 10^{-5} \cdot(T / \mathrm{K})+4.34582 \cdot 10^{-8} \cdot(T / \mathrm{K})^{2}, B=-1.26727$. $10^{3}+8.06157 \cdot 10^{1} \cdot(T / \mathrm{K})-1.26389 \cdot 10^{-1} \cdot(T / \mathrm{K})^{2}$.

values are within acceptable limits, they corroborate the strong dependence of the derivation method on the shape of the curves used to fit the experimental data and the necessity of collecting a large number of experimental points.

As for the isobaric heat capacity, results reveal a decrease with increasing pressure contrarily to the pressure

TABLE 11

Experimental density, $\rho /\left(\mathrm{kg} \cdot \mathrm{m}^{-3}\right)$, data for $[\mathrm{hmim}]\left[\mathrm{NTf}_{2}\right]$ as a function of temperature, $T$, and pressure, $p$

\begin{tabular}{|c|c|c|c|c|c|c|c|c|}
\hline \multirow[t]{2}{*}{$p / \mathrm{MPa}$} & \multicolumn{8}{|l|}{$T / \mathrm{K}$} \\
\hline & 298.15 & 299.15 & 300.15 & 301.15 & 302.15 & 303.15 & 308.15 & 313.15 \\
\hline 0.10 & 1370.81 & 1369.96 & 1368.97 & 1368.11 & 1367.15 & 1366.24 & 1361.65 & 1357.01 \\
\hline 1.83 & 1372.13 & 1371.27 & 1370.29 & 1369.42 & 1368.47 & 1367.56 & 1362.96 & 1358.32 \\
\hline 10.02 & 1378.18 & 1377.30 & 1376.36 & 1375.49 & 1374.56 & 1373.64 & 1369.05 & 1364.45 \\
\hline 14.98 & 1381.70 & 1380.81 & 1379.86 & 1379.01 & 1378.10 & 1377.17 & 1372.62 & 1368.01 \\
\hline 19.75 & 1384.98 & 1384.12 & 1383.21 & 1382.28 & 1381.43 & 1380.47 & 1375.95 & 1371.36 \\
\hline 25.86 & 1389.10 & 1388.22 & 1387.32 & 1386.44 & 1385.56 & 1384.59 & 1380.12 & 1375.57 \\
\hline 31.48 & 1392.73 & 1391.88 & 1391.00 & 1390.11 & 1389.24 & 1388.30 & 1383.86 & 1379.34 \\
\hline 37.77 & 1396.74 & 1395.86 & 1395.01 & 1394.07 & 1393.28 & & 1387.93 & 1383.47 \\
\hline 44.17 & 1400.64 & 1399.77 & 1398.88 & 1398.06 & 1397.22 & 1396.31 & 1391.98 & 1387.60 \\
\hline 50.75 & 1404.48 & 1403.70 & 1402.84 & 1401.99 & 1401.19 & 1400.29 & 1396.05 & 1391.76 \\
\hline 59.59 & 1409.53 & 1408.77 & 1407.93 & 1407.14 & 1406.37 & 1405.51 & 1401.40 & 1397.25 \\
\hline
\end{tabular}

\begin{tabular}{|c|c|c|c|c|c|c|c|}
\hline & \multicolumn{7}{|l|}{$T / \mathrm{K}$} \\
\hline & 318.15 & 323.15 & 325.15 & 327.15 & 329.15 & 331.15 & 333.15 \\
\hline 0.10 & 1352.32 & 1348.05 & 1346.34 & 1344.39 & 1342.33 & 1340.37 & 1338.24 \\
\hline 1.83 & 1353.62 & 1349.36 & 1347.70 & 1345.74 & 1343.71 & 1341.75 & 1339.65 \\
\hline 10.02 & 1359.84 & 1355.55 & 1354.03 & 1352.05 & 1350.14 & 1348.20 & 1346.20 \\
\hline 14.98 & 1363.45 & 1359.13 & 1357.71 & 1355.75 & 1353.87 & 1351.91 & 1349.99 \\
\hline 19.75 & & 1362.50 & 1361.18 & 1359.21 & 1357.38 & 1355.44 & 1353.57 \\
\hline 25.86 & 1371.10 & 1366.73 & 1365.48 & 1363.58 & 1361.77 & 1359.84 & 1358.01 \\
\hline 31.48 & 1374.91 & 1370.56 & 1369.35 & 1367.53 & 1365.68 & 1363.79 & 1361.98 \\
\hline 37.77 & 1379.09 & 1374.72 & 1373.57 & 1371.82 & 1369.99 & 1368.10 & 1366.34 \\
\hline 44.17 & 1383.28 & 1378.88 & 1377.76 & 1376.09 & 1374.26 & 1372.39 & 1370.63 \\
\hline 50.75 & 1387.51 & 1383.17 & 1382.02 & 1380.39 & 1378.59 & 1376.72 & 1374.95 \\
\hline 59.59 & 1393.09 & 1388.84 & 1387.57 & 1386.07 & 1384.28 & 1382.44 & 1380.65 \\
\hline
\end{tabular}


TABLE 13

Parameters of the linear fit $\ln \left(\rho /\left(\mathrm{kg} \cdot \mathrm{m}^{-3}\right)\right)=c+d \cdot(T / \mathrm{K})$ of $[\mathrm{hmim}]\left[\mathrm{NTf}_{2}\right]$

\begin{tabular}{cll}
\hline$p / \mathrm{MPa}$ & $c$ & $d\left(\mathrm{~K}^{-1}\right) \cdot 10^{4}$ \\
\hline 0.10 & 7.4257 & -6.7918 \\
1.83 & 7.4260 & -6.7705 \\
10.02 & 7.4269 & -6.6515 \\
14.98 & 7.4274 & -6.5846 \\
19.75 & 7.4277 & -6.5147 \\
25.86 & 7.4282 & -6.4340 \\
31.48 & 7.4284 & -6.3514 \\
37.77 & 7.4285 & -6.2597 \\
44.17 & 7.4283 & -6.1580 \\
50.75 & 7.4277 & -6.0448 \\
59.59 & 7.4264 & -5.8811 \\
\hline
\end{tabular}

independence observed in $[\mathrm{bmim}]\left[\mathrm{PF}_{6}\right]$ and $[\mathrm{bmim}]\left[\mathrm{BF}_{4}\right]$ [16]. This is due both to the expansivity and its temperature dependence as can be judged by the analysis of equation (6).

\section{2. $[\mathrm{hmim}]\left[N T f_{2}\right]$}

Density measurements have been carried out at a broad range of temperatures $(298<T / \mathrm{K}<333)$ and pressures $(0.1<p / \mathrm{MPa}<60)$. The experimental data are presented both in figure 9 and table 11. At atmo- spheric pressure, our results are about $0.06 \%$ below those presented by Canongia Lopes et al. [14].

The absence of heat capacity and speed of sound data compelled the direct use of the derivatives of density in order to obtain the derived thermodynamic properties of this substance, namely, $\alpha_{p}, \kappa_{T}$ and $\gamma_{v}$. The fitting of isobaric density data was performed through the Tait equation as presented in equation (7). This equation is known to represent very well the density behaviour of liquids over pressure at constant temperature

$\frac{1}{\rho}=\frac{1}{\rho^{*}}+A \cdot \ln \left(\frac{B+p}{B+0.1}\right)$.

The parameters for each isotherm are shown in table 12. The isothermal compressibility is calculated using the isothermal pressure derivative of density according to the following equation:

$\kappa_{T}=\frac{1}{\rho}\left(\frac{\partial \rho}{\partial p}\right)_{T}$.

Unfortunately, there is no equivalent of the Tait equation for the behaviour of density with temperature at constant pressure. A detailed evaluation of the raw data reveals that deviations from linearity in densitytemperature (or volume-temperature) plots are so mild that the determination of the temperature coefficient of $\alpha_{p}$ is extremely dependent on the choice of the type of

TABLE 14

Calculated values of $\kappa_{T} l\left(\mathrm{GPa}^{-1}\right)$ for $[\mathrm{hmim}]\left[\mathrm{NTf}_{2}\right]$

\begin{tabular}{|c|c|c|c|c|c|c|c|c|}
\hline \multirow[t]{2}{*}{$p / \mathrm{MPa}$} & \multicolumn{8}{|l|}{$T / \mathrm{K}$} \\
\hline & 298.15 & 299.15 & 300.15 & 301.15 & 302.15 & 303.15 & 308.15 & 313.15 \\
\hline 0.10 & $0.561_{3}$ & $0.560_{1}$ & $0.559_{1}$ & $0.558_{4}$ & $0.557_{8}$ & $0.557_{3}$ & $0.557_{1}$ & $0.559_{8}$ \\
\hline 1.83 & $0.554_{4}$ & $0.553_{5}$ & $0.552_{8}$ & $0.552_{2}$ & $0.551_{8}$ & $0.551_{5}$ & $0.552_{0}$ & $0.554_{9}$ \\
\hline 10.02 & $0.523_{7}$ & $0.524_{0}$ & $0.524_{4}$ & $0.524_{8}$ & $0.525_{3}$ & $0.525_{8}$ & $0.528_{9}$ & $0.533_{1}$ \\
\hline 14.98 & $0.506_{8}$ & $0.507_{8}$ & $0.508_{7}$ & $0.509_{6}$ & $0.510_{5}$ & $0.511_{4}$ & $0.515_{9}$ & $0.520_{8}$ \\
\hline 19.75 & $0.491_{6}$ & $0.493_{1}$ & $0.494_{5}$ & $0.495_{8}$ & $0.497_{1}$ & $0.498_{3}$ & $0.503_{9}$ & $0.509_{5}$ \\
\hline 25.86 & $0.473_{4}$ & $0.475_{5}$ & $0.477_{4}$ & $0.479_{2}$ & $0.480_{9}$ & $0.482_{5}$ & $0.489_{5}$ & $0.495_{8}$ \\
\hline 31.48 & $0.457_{9}$ & $0.460_{5}$ & $0.462_{8}$ & $0.464_{9}$ & $0.467_{0}$ & $0.468_{8}$ & $0.477_{0}$ & $0.483_{8}$ \\
\hline 37.77 & $0.441_{8}$ & $0.444_{7}$ & $0.447_{5}$ & $0.450_{0}$ & $0.452_{4}$ & & $0.463_{7}$ & $0.471_{1}$ \\
\hline 44.17 & $0.426_{5}$ & $0.429_{9}$ & $0.433_{0}$ & $0.435_{8}$ & $0.438_{5}$ & $0.440_{9}$ & $0.451_{0}$ & $0.458_{9}$ \\
\hline 50.75 & $0.411_{9}$ & $0.415_{6}$ & $0.419_{0}$ & $0.422_{1}$ & $0.425_{0}$ & $0.427_{7}$ & $0.438_{7}$ & $0.447_{0}$ \\
\hline 59.59 & $0.393_{9}$ & $0.398_{0}$ & $0.401_{7}$ & $0.405_{1}$ & $0.408_{3}$ & $0.411_{3}$ & $0.423_{3}$ & $0.432_{1}$ \\
\hline
\end{tabular}

\begin{tabular}{|c|c|c|c|c|c|c|c|}
\hline & \multicolumn{7}{|l|}{$T / \mathrm{K}$} \\
\hline & 318.15 & 323.15 & 325.15 & 327.15 & 329.15 & 331.15 & 333.15 \\
\hline 0.10 & $0.565_{2}$ & $0.574_{2}$ & $0.579_{0}$ & $0.584_{6}$ & $0.591_{1}$ & $0.599_{0}$ & $0.608_{3}$ \\
\hline 1.83 & $0.560_{5}$ & $0.569_{3}$ & $0.574_{0}$ & $0.579_{4}$ & $0.585_{8}$ & $0.593_{3}$ & $0.602_{3}$ \\
\hline 10.02 & $0.539_{0}$ & $0.547_{3}$ & $0.551_{5}$ & $0.556_{2}$ & $0.561_{6}$ & $0.568_{0}$ & $0.575_{5}$ \\
\hline 14.98 & $0.526_{9}$ & $0.534_{8}$ & $0.538_{8}$ & $0.543_{1}$ & $0.548_{0}$ & $0.553_{7}$ & $0.560_{4}$ \\
\hline 19.75 & & $0.523_{3}$ & $0.527_{1}$ & $0.531_{0}$ & $0.535_{6}$ & $0.540_{7}$ & $0.546_{7}$ \\
\hline 25.86 & $0.502_{1}$ & $0.509_{4}$ & $0.512_{8}$ & $0.516_{4}$ & $0.520_{5}$ & $0.525_{0}$ & $0.530_{2}$ \\
\hline 31.48 & $0.490_{3}$ & $0.497_{2}$ & $0.500_{4}$ & $0.503_{7}$ & $0.507_{3}$ & $0.511_{3}$ & $0.515_{8}$ \\
\hline 37.77 & $0.477_{7}$ & $0.484_{3}$ & $0.487_{3}$ & $0.490_{3}$ & $0.493_{4}$ & $0.496_{9}$ & $0.500_{7}$ \\
\hline 44.17 & $0.465_{6}$ & $0.471_{9}$ & $0.474_{7}$ & $0.477_{4}$ & $0.480_{1}$ & $0.483_{1}$ & $0.486_{3}$ \\
\hline 50.75 & $0.453_{8}$ & $0.459_{9}$ & $0.462_{4}$ & $0.464_{8}$ & $0.467_{2}$ & $0.469_{7}$ & $0.472_{4}$ \\
\hline 59.59 & $0.439_{0}$ & $0.444_{7}$ & $0.447_{0}$ & $0.449_{0}$ & $0.451_{0}$ & $0.452_{9}$ & $0.454_{9}$ \\
\hline
\end{tabular}


TABLE 15

Calculated values of $\gamma_{v} /\left(\mathrm{MPa} \cdot \mathrm{K}^{-1}\right)$ for $[\mathrm{hmim}]\left[\mathrm{NTf}_{2}\right]$

\begin{tabular}{|c|c|c|c|c|c|c|c|c|}
\hline \multirow[t]{2}{*}{$p / \mathrm{MPa}$} & \multicolumn{8}{|l|}{$T / \mathrm{K}$} \\
\hline & 298.15 & 299.15 & 300.15 & 301.15 & 302.15 & 303.15 & 308.15 & 313.15 \\
\hline 0.10 & $1.21_{0}$ & $1.21_{3}$ & $1.21_{5}$ & $1.21_{6}$ & $1.21_{8}$ & $1.21_{9}$ & $1.21_{9}$ & $1.21_{3}$ \\
\hline 1.83 & $1.22_{1}$ & $1.22_{3}$ & $1.22_{5}$ & $1.22_{6}$ & $1.22_{7}$ & $1.22_{8}$ & $1.22_{7}$ & $1.22_{0}$ \\
\hline 10.02 & $1.27_{0}$ & $1.26_{9}$ & $1.26_{8}$ & $1.26_{7}$ & $1.26_{6}$ & $1.26_{5}$ & $1.25_{8}$ & $1.24_{8}$ \\
\hline 14.98 & $1.29_{9}$ & $1.29_{7}$ & $1.29_{4}^{\circ}$ & $1.29_{2}$ & $1.29_{0}$ & $1.28_{8}$ & $1.27_{6}$ & $1.26_{4}$ \\
\hline 19.75 & $1.32_{5}$ & $1.32_{1}$ & $1.31_{8}$ & $1.31_{4}$ & $1.31_{1}$ & $1.30_{7}$ & $1.29_{3}$ & $1.27_{9}$ \\
\hline 25.86 & $1.35_{9}$ & $1.35_{3}$ & $1.34_{8}$ & $1.34_{3}$ & $1.33_{8}$ & $1.33_{4}$ & $1.31_{4}$ & $1.29_{8}$ \\
\hline 31.48 & $1.38_{7}$ & $1.37_{9}$ & $1.37_{2}$ & $1.36_{6}$ & $1.36_{0}$ & $1.35_{5}$ & $1.33_{2}$ & $1.31_{3}$ \\
\hline 37.77 & $1.41_{7}$ & $1.40_{7}$ & 1.399 & $1.39_{1}$ & $1.38_{4}$ & & $1.35_{0}$ & $1.32_{9}$ \\
\hline 44.17 & $1.44_{4}$ & $1.43_{2}$ & $1.42_{2}$ & $1.41_{3}$ & $1.40_{4}$ & $1.39_{7}$ & $1.36_{5}$ & $1.34_{2}$ \\
\hline 50.75 & $1.46_{7}$ & $1.45_{4}$ & $1.44_{3}$ & $1.43_{2}$ & $1.42_{2}$ & $1.41_{3}$ & $1.37_{8}$ & $1.35_{2}$ \\
\hline 59.59 & $1.49_{3}$ & $1.47_{8}$ & $1.46_{4}$ & $1.45_{2}$ & $1.44_{0}$ & $1.43_{0}$ & $1.38_{9}$ & $1.36_{1}$ \\
\hline
\end{tabular}

\begin{tabular}{rlllllll}
\multicolumn{1}{c}{$T / \mathrm{K}$} & \multicolumn{7}{l}{} \\
\hline & 318.15 & 323.15 & 325.15 & 327.15 & 329.15 & 331.15 & 333.15 \\
\hline 0.10 & $1.20_{2}$ & $1.18_{3}$ & $1.17_{3}$ & $1.16_{2}$ & $1.14_{9}$ & $1.13_{4}$ & $1.11_{7}$ \\
1.83 & $1.20_{8}$ & $1.18_{9}$ & $1.17_{9}$ & $1.16_{8}$ & $1.15_{6}$ & $1.12_{4}$ \\
10.02 & $1.23_{4}$ & $1.21_{5}$ & $1.20_{6}$ & $1.19_{6}$ & $1.18_{4}$ & $1.17_{1}$ & $1.15_{6}$ \\
14.98 & $1.25_{0}$ & $1.23_{1}$ & $1.22_{2}$ & $1.21_{2}$ & $1.20_{2}$ & $1.18_{9}$ & $1.17_{5}$ \\
19.75 & & $1.24_{5}$ & $1.23_{6}$ & $1.22_{7}$ & $1.21_{6}$ & $1.19_{2}$ \\
25.86 & $1.28_{1}$ & $1.26_{3}$ & $1.25_{5}$ & $1.24_{6}$ & $1.23_{6}$ & $1.22_{6}$ & $1.21_{4}$ \\
31.48 & $1.29_{5}$ & $1.27_{7}$ & $1.26_{9}$ & $1.26_{1}$ & $1.25_{2}$ & $1.24_{2}$ & $1.23_{1}$ \\
37.77 & $1.31_{0}$ & $1.29_{3}$ & $1.28_{5}$ & $1.27_{7}$ & $1.26_{9}$ & $1.26_{0}$ & $1.25_{0}$ \\
44.17 & $1.32_{3}$ & $1.30_{5}$ & $1.29_{7}$ & $1.29_{0}$ & $1.28_{3}$ & $1.27_{5}$ & $1.26_{6}$ \\
50.75 & $1.33_{2}$ & $1.31_{4}$ & $1.30_{7}$ & $1.30_{0}$ & $1.29_{4}$ & $1.28_{7}$ & $1.28_{0}$ \\
59.59 & $1.34_{0}$ & $1.32_{2}$ & $1.31_{6}$ & $1.31_{0}$ & $1.30_{4}$ & $1.29_{8}$ & $1.29_{3}$ \\
\hline
\end{tabular}

function to screen the data. For those situations where the statistical scatter of the raw data is large compared with an unambiguous determination of the curvature, we suggest [16] the use of $\ln \rho=f(T)$. If this function proves to be linear, then, $\ln V=f(T)$ is as well, and $\alpha_{p}$ is constant (temperature independent). Moreover, the choice of a logarithmic function avoids the existence of a mathematical violation which arises if one considers

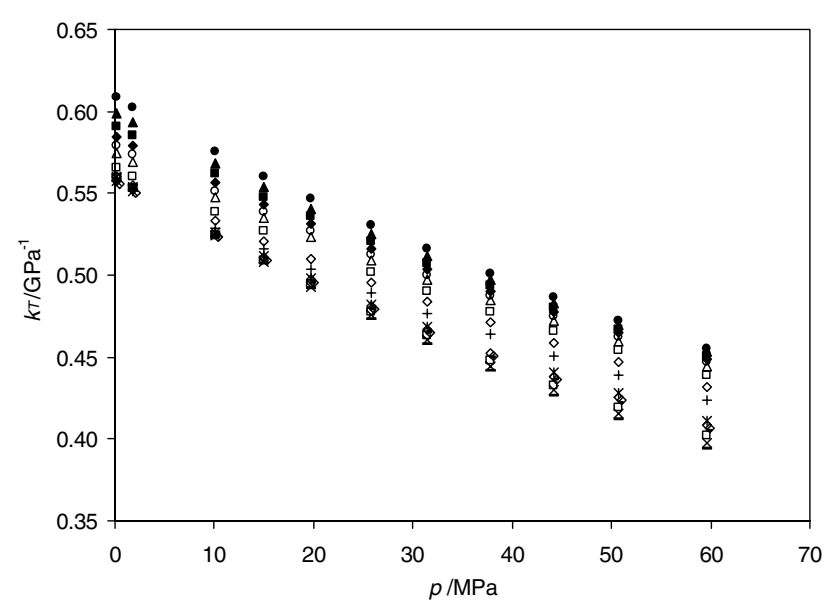

FIGURE 10. Isotherms for the isobaric expansivity of [hmim][NTf $\left.{ }_{2}\right]$. 一, $298.15 \mathrm{~K} ; \times, 299.15 \mathrm{~K}$; 叀 $300.15 \mathrm{~K} ;--, 301.15 \mathrm{~K} ; \diamond, 302.15 \mathrm{~K} ; \boldsymbol{*}$, $303.15 \mathrm{~K} ;+, 308.15 \mathrm{~K} ; \diamond, 313.15 \mathrm{~K} ; \square, 318.15 \mathrm{~K} ; \triangle, 323.15 \mathrm{~K} ; \bigcirc$, $325.15 \mathrm{~K} ; \bullet, 327.15 \mathrm{~K} ; \mathbf{\square}, 329.15 \mathrm{~K} ; \boldsymbol{\Delta}, 331.15 \mathrm{~K} ; \bullet$ ๑, 333.15 K. that both $\rho-T$ and $V-T$ plots are linear. For [hmim]$\left[\mathrm{NTF}_{2}\right]$ the behaviour of $\ln \rho$ proved to be linear and the coefficients of the fitting are presented on table 13 .

The calculated properties, such as $\kappa_{T}, \alpha_{p}$ and $\gamma_{v}$ and are presented in tables 14 and 15 and figures 10 to 12 . As far as the authors are aware of, there are no other data available for comparison of the properties of $[\mathrm{hmim}]\left[\mathrm{NTF}_{2}\right]$.

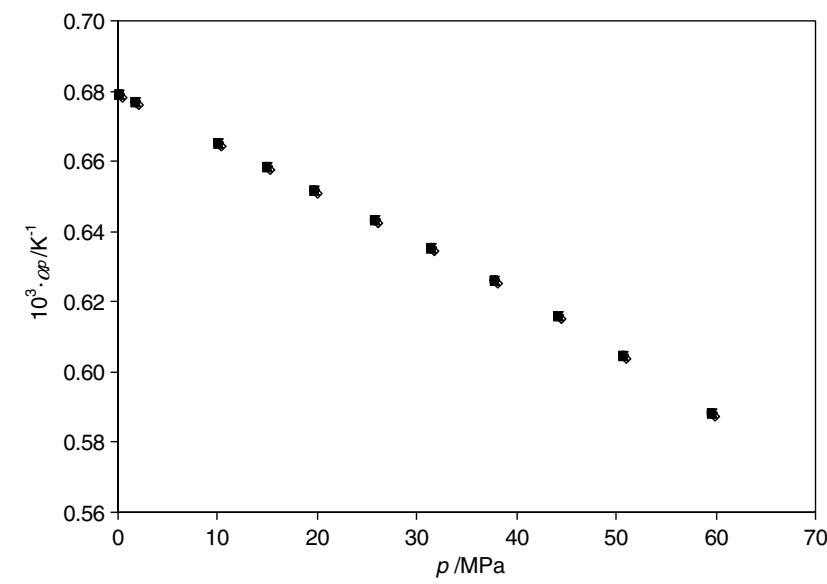

FIGURE 11. Isotherms for the isothermal compressibility of [hmim][NTf ${ }_{2}$ ].-, 298.15 K; ×, 299.15 K; 本, 300.15 K; -, 301.15 K; $\diamond, 302.15 \mathrm{~K}$; *, $303.15 \mathrm{~K} ;+, 308.15 \mathrm{~K} ; \diamond, 313.15 \mathrm{~K} ; \square, 318.15 \mathrm{~K} ; \triangle, 323.15 \mathrm{~K} ; \bigcirc$, $325.15 \mathrm{~K} ; \bullet, 327.15 \mathrm{~K} ; \boldsymbol{\square}, 329.15 \mathrm{~K} ; \boldsymbol{\Delta}, 331.15 \mathrm{~K} ; \bullet, 333.15 \mathrm{~K}$. Since $\alpha_{p}$ is $T$-independent all symbols overlap - see text. 


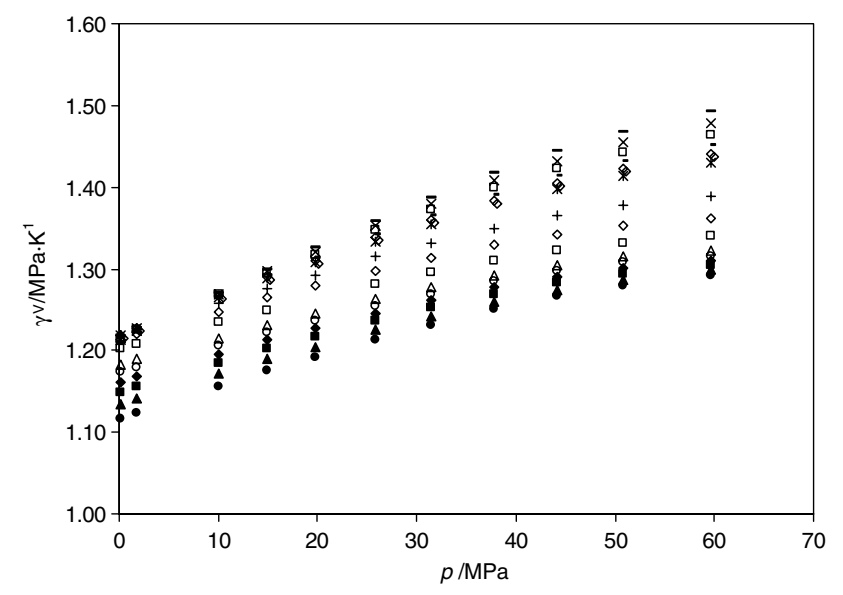

FIGURE 12. Isotherms for the thermal pressure coefficient of [hmim][NTf ${ }_{2}$ ]. 一, $298.15 \mathrm{~K} ; \times, 299.15 \mathrm{~K}$; 屯, $300.15 \mathrm{~K} ;--, 301.15 \mathrm{~K} ; \diamond, 302.15$ $\mathrm{K} ; \boldsymbol{*}, 303.15 \mathrm{~K} ;+, 308.15 \mathrm{~K} ; \diamond, 313.15 \mathrm{~K} ; \square, 318.15 \mathrm{~K} ; \triangle, 323.15 \mathrm{~K}$;

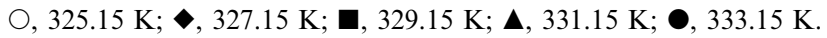

\section{Conclusions}

The work performed intends to map the thermophysical and thermodynamic behaviour of two important ionic liquids over wide pressure and temperature ranges.

The increase of the alkyl chain of the 1-alkyl-3methylimidazolium cation results in a decrease in the density. This fact has also been reported in other works $[1-3,13,14]$. This trend is precisely the opposite of the effect of an increase in the anion size [1-3,16]. It should be noted that while in the first case one witnesses density changes upon different alkyl substitutions within the same "cation's family", in the second case, the anions belong to distinct chemical species. The comparison with some literature data [16] allows us to state that a change from $[\mathrm{bmim}]\left[\mathrm{NTF}_{2}\right]$ to $[\mathrm{hmim}]\left[\mathrm{NTF}_{2}\right]$ shows almost the same effect on density as the change from [bmim $]\left[\mathrm{NTF}_{2}\right]$ to $[\mathrm{bmim}]\left[\mathrm{PF}_{6}\right]$. However, this change in density is not accompanied by a similar effect on $\alpha_{p}$ and $\kappa_{T}$. Here, the alkyl chain effect of the cation is very mild, and it is found that pressure effects are significant. When density data is translated to molar volume values, one finds that the addition of two $\left(\mathrm{CH}_{2}\right)$ units on changing from $[\mathrm{bmim}]^{+}$to $[\mathrm{hmim}]^{+}$in $\left[\mathrm{Ntf}_{2}\right]^{-}$-based ILs provokes, at $298.15 \mathrm{~K}$ and atmospheric pressure, a molar volume increase of $34.56 \cdot 10^{-6} \mathrm{~m}^{3} \cdot \mathrm{mol}^{-1}$. This experimental result corroborates previous estimations [1] that established a value of $(34.4 \pm 0.5) \cdot 10^{-6} \mathrm{~m}^{3} \cdot \mathrm{mol}^{-1}$ for this same effect irrespective of the counter-anion considered.

The data obtained confirm the prediction of the effect of the anion on both the speed of sound and density. As the anions of different chemical origin increase in size $\left(\left[\mathrm{BF}_{4}\right]^{-}<\left[\mathrm{PF}_{6}\right]^{-}<\left[\mathrm{NTf}_{2}\right]^{-}\right)$the density increases accordingly, while the sound-speed shows the opposite trend. As previously stated $[1,16]$, compressibility is the factor which controls the sound-speed. This statement is also well illustrated by analyzing the variation of these three quantities as pressure is applied. Note that, as pressure rises, density increases, compressibility decreases, and the sound-speed increases.

The effect of pressure on the heat capacities of $[\mathrm{bmim}]\left[\mathrm{NTf}_{2}\right]$ is greater than that on [16] $[\mathrm{bmim}]\left[\mathrm{PF}_{6}\right]$ and $[\mathrm{bmim}]\left[\mathrm{BF}_{4}\right]$ which is explained by the higher temperature dependence of the isobaric expansivity. If comparisons are performed using molar quantities, for instance, at atmospheric pressure and $298.15 \mathrm{~K}, C_{\mathrm{PbmimNTf}_{2}}-C_{\mathrm{PbmimPF}_{6}}=177 \mathrm{~J} \cdot \mathrm{mol}^{-1} \cdot \mathrm{K}^{-1}$ and $C_{\mathrm{PbmimNTf}_{2}}-C_{\mathrm{PbmimBF}_{4}}=221.1 \mathrm{~J} \cdot \mathrm{mol}^{-1} \cdot \mathrm{K}^{-1}$. The greater values presented by $[\mathrm{bmim}]\left[\mathrm{NTf}_{2}\right]$ are mainly a consequence of the larger number of vibrational degrees of freedom of this anion compared with those of $[\mathrm{bmim}]\left[\mathrm{PF}_{6}\right]$ and $[\mathrm{bmim}]\left[\mathrm{BF}_{4}\right]$. In other words, as the anion's structural complexity increases one expects improved ability for energy storage. In the case of an increase in the alkyl chain length of the cation, one also expects an increase in $C_{p}$. For instance, $[\mathrm{hmim}]\left[\mathrm{NTf}_{2}\right]$ has $3 \cdot 6=18$ internal, vibrational degrees of freedom in excess to those presented by [bmim] $\left[\mathrm{NTf}_{2}\right]$.

\section{Acknowledgements}

This work was financially supported by Fundação para a Ciência e Tecnologia, Portugal, under contract POCTI/EQU/35437/00. R.G.A., J.M.S.S.E., and Z.P.V. are grateful to Fundação para a Ciência e Tecnologia for doctoral fellowships. The authors wish to thank the QUILL group, Belfast, led by Prof. Kenneth Seddon for hosting some of us and helping on the synthesis and purification of both ionic liquids.

\section{References}

[1] L.P.N. Rebelo, V. Najdanovic-Visak, R. Gomes de Azevedo, J.M.S.S. Esperança, M. Nunes da Ponte, H.J.R. Guedes, Z.P. Visak, H.C. de Sousa, J. Szydlowski, J.N. Canongia Lopes, T.C. Cordeiro, in: R. Rogers, K.R. Seddon (Eds.), Ionic Liquids IIIA: Fundamentals, Progress, Challenges, and Opportunities - Properties and Structure, ACS Symposium Series, vol. 901, American Chemical Society, Whashington, DC, 2005, pp. 260-281 (Chapter 21).

[2] C.P. Fredlake, J.M. Crosthwaite, D.G. Hert, S.N.V.K. Aki, J.F. Brennecke, J. Chem. Eng. Data 49 (2004) 954-964.

[3] (a) H. Tokuda, K. Hayamizu, K. Ishii, M.A.B.H. Susan, M. Watanabe, J. Phys. Chem. B 108 (2004) 16593-16600;

(b) H. Tokuda, K. Hayamizu, K. Ishii, M.A.B.H. Susan, M. Watanabe, J. Phys. Chem. B 109 (2005) 6103-6110.

[4] K.N. Marsh, J.A. Boxall, R. Lichtenthaler, Fluid Phase Equilibr. 219 (2004) 93-98.

[5] R. Gomes de Azevedo, J. Szydlowski, P.F. Pires, J.M.S.S. Esperança, H.J.R. Guedes, L.P.N. Rebelo, J. Chem. Thermodyn. 36 (2004) 211-222. 
[6] J. Szydlowski, R. Gomes de Azevedo, L.P.N. Rebelo, J.M.S.S Esperança, H.J.R. Guedes, J. Chem. Thermodyn. 37 (2005) 671-683.

[7] J.P.M. Trusler, Physical acoustics and metrology of fluids, Adam Hilger, Bristol, 1991.

[8] G. Douhéret, M.I. Davis, J.C.R. Reis, M.J. Blandamer, ChemPhysChem 2 (2001) 148-161.

[9] P.F. Pires, J.M.S.S. Esperança, H.J.R. Guedes, J. Chem. Eng. Data 45 (2000) 496-502.

[10] J.L. Daridon, A. Lagrabette, B. Lagourette, J. Chem. Thermodyn. 30 (1998) 607-623.

[11] P. Bonhôte, A.-P. Dias, M. Armand, N. Papageorgiou, K. Kalyanasundaram, M. Gratzel, Inorg. Chem. 35 (1996) 1168-1178.

[12] J.D. Holbrey, W.M. Reichert, R.G. Reddy, R.D. Rogers, in: K.R. Seddon, R.D. Rogers (Eds.), Ionic Liquids as Green
Solvents, ACS symposium series, vol. 856, American Chemical Society, Washington, DC, 2003, pp. 121-133.

[13] S.V. Dzyuba, R.A. Bartsch, ChemPhysChem 3 (2002) 161166.

[14] J.N. Canongia Lopes, T.C. Cordeiro, J.M.S.S. Esperança, H.J.R. Guedes, S. Huk, L.P.N. Rebelo, K.R. Seddon, J. Phys. Chem. B 109 (2005) 3519-3525.

[15] T. Sun, S.N. Biswas, N.J. Trappeniers, C.A.T. Seldam, J. Chem. Eng. Data 33 (1988) 395-398.

[16] R. Gomes de Azevedo, J.M.S.S. Esperança, V. Najdanovic-Visak, Z.P. Visak, H.J.R. Guedes, M. Nunes da Ponte, L.P.N. Rebelo, J. Chem. Eng. Data 50 (2005) 997-1008.

\section{JCT 05-22}

